\title{
Nuclear genome sequence of the plastid- lacking cryptomonad Goniomonas avonlea provides insights into the evolution of secondary plastids
}

Ugo Cenci ${ }^{1,2+}$, Shannon J. Sibbald ${ }^{1,2+}$, Bruce A. Curtis ${ }^{1,2}$, Ryoma Kamikawa ${ }^{3}$, Laura Eme ${ }^{1,2,11}$, Daniel Moog ${ }^{1,2,12}$, Bernard Henrissat ${ }^{4,5,6}$, Eric Maréchal ${ }^{7}$, Malika Chabi ${ }^{8}$, Christophe Djemiel ${ }^{8}$, Andrew J. Roger ${ }^{1,2,9}$, Eunsoo Kim ${ }^{10}$ and John M. Archibald ${ }^{1,2,9^{*}}$ (i)

\begin{abstract}
Background: The evolution of photosynthesis has been a major driver in eukaryotic diversification. Eukaryotes have acquired plastids (chloroplasts) either directly via the engulfment and integration of a photosynthetic cyanobacterium (primary endosymbiosis) or indirectly by engulfing a photosynthetic eukaryote (secondary or tertiary endosymbiosis). The timing and frequency of secondary endosymbiosis during eukaryotic evolution is currently unclear but may be resolved in part by studying cryptomonads, a group of single-celled eukaryotes comprised of both photosynthetic and non-photosynthetic species. While cryptomonads such as Guillardia theta harbor a red algal-derived plastid of secondary endosymbiotic origin, members of the sister group Goniomonadea lack plastids. Here, we present the genome of Goniomonas avonlea - the first for any goniomonad - to address whether Goniomonadea are ancestrally non-photosynthetic or whether they lost a plastid secondarily.

Results: We sequenced the nuclear and mitochondrial genomes of Goniomonas avonlea and carried out a comparative analysis of Go. avonlea, Gu. theta, and other cryptomonads. The Go. avonlea genome assembly is $\sim 92$ Mbp in size, with 33,470 predicted protein-coding genes. Interestingly, some metabolic pathways (e.g., fatty acid biosynthesis) predicted to occur in the plastid and periplastidal compartment of $\mathrm{Gu}$. theta appear to operate in the cytoplasm of Go. avonlea, suggesting that metabolic redundancies were generated during the course of secondary plastid integration. Other cytosolic pathways found in Go. avonlea are not found in Gu. theta, suggesting secondary loss in Gu. theta and other plastid-bearing cryptomonads. Phylogenetic analyses revealed no evidence for algal endosymbiont-derived genes in the Go. avonlea genome. Phylogenomic analyses point to a specific relationship between Cryptista (to which cryptomonads belong) and Archaeplastida.

Conclusion: We found no convincing genomic or phylogenomic evidence that Go. avonlea evolved from a secondary red algal plastid-bearing ancestor, consistent with goniomonads being ancestrally non-photosynthetic eukaryotes. The Go. avonlea genome sheds light on the physiology of heterotrophic cryptomonads and serves as an important reference point for studying the metabolic "rewiring" that took place during secondary plastid integration in the ancestor of modern-day Cryptophyceae.
\end{abstract}

Keywords: Cryptomonads, Cryptophytes, Secondary endosymbiosis, Phylogenomics, Genome evolution

\footnotetext{
* Correspondence: john.archibald@dal.ca

†Ugo Cenci and Shannon J. Sibbald contributed equally to this work.

${ }^{1}$ Department of Biochemistry and Molecular Biology, Dalhousie University,

Halifax, Nova Scotia B3H 4R2, Canada

${ }^{2}$ Centre for Comparative Genomics and Evolutionary Bioinformatics,

Dalhousie University, Halifax, Nova Scotia, Canada

Full list of author information is available at the end of the article
}

(c) The Author(s). 2018 Open Access This article is distributed under the terms of the Creative Commons Attribution 4.0 International License (http://creativecommons.org/licenses/by/4.0/), which permits unrestricted use, distribution, and reproduction in any medium, provided you give appropriate credit to the original author(s) and the source, provide a link to the Creative Commons license, and indicate if changes were made. The Creative Commons Public Domain Dedication waiver (http://creativecommons.org/publicdomain/zero/1.0/) applies to the data made available in this article, unless otherwise stated. 


\section{Background}

The acquisition of photosynthesis in eukaryotes can be traced back to a primary endosymbiosis in which a eukaryotic host engulfed and assimilated a photosynthetic cyanobacterium, which ultimately became the plastid (chloroplast) [1, 2]. Canonical "primary" plastids are surrounded by two membranes and are generally thought to have evolved on a single occasion in the common ancestor of Archaeplastida, a tripartite eukaryotic "supergroup" comprised of Viridiplantae (also known as Chloroplastida), Rhodophyta (Rhodophyceae), and Glaucophyta [3-5]. Eukaryotes have also acquired photosynthesis indirectly on multiple occasions via "secondary" (i.e., eukaryote-eukaryote) endosymbiosis. Indeed, secondary (and in some cases tertiary) endosymbiosis is thought to have given rise to plastids scattered amongst the stramenopiles, alveolates, rhizarians, euglenozoans, haptophytes, and cryptomonads [6]. The latter lineage is divided into two clades, the plastid-bearing, mostly photosynthetic Cryptophyceae and the heterotrophic Goniomonadea. The evolutionary distinctness of these two clades makes for an interesting case study with which to understand the transition from a plastid-lacking eukaryote to a photosynthetic, secondary plastid-bearing organism.

Guillardia theta and the recently described Goniomonas avonlea [7] are representatives of plastid-bearing and plastid-lacking cryptomonads [5, 8], respectively. Together with several paraphyletic plastid-lacking lineages, including katablepharids and Palpitomonas, cryptomonads constitute a clade known as Cryptista $[9,10]$. The position of Cryptista on the eukaryotic tree of life is a point of contention. Some phylogenomic studies have placed it sister to Haptophyta (e.g., [11]), with the Cryptista-Haptophyta clade itself branching either next to the SAR supergroup (Stramenopiles, Alveolata, Rhizaria; e.g., [12]) or the Archaeplastida (e.g., [13]). Other studies have suggested that Cryptista and Haptophyta are not specifically related, with the former branching within the Archaeplastida [14]. Our knowledge of Cryptista and their evolutionary history has suffered from a paucity of genomic data [15]. Only one species, $G u$. theta, which has been studied mainly for its plastid and nucleomorph (the vestigial nucleus of the red alga acquired by secondary endosymbiosis) $[16,17]$ has had its nuclear genome sequenced [18]. The diversity of plastid-lacking species within cryptomonads and, more broadly, Cryptista, has received relatively little attention.

The transition from a heterotrophic, aplastidic cell to a plastid-bearing one is associated with the acquisition of a wide range of metabolic capabilities, such as photosynthesis and novel amino acid biosynthetic capacities [19]. The acquisition of photosynthesis and carbon fixation by a heterotrophic protist impacts the regulation of many of its metabolic pathways [20]. In addition, pathways operating in different subcellular compartments can become partially or completely redundant. This allows the organism to tinker with the regulation of pathways that may be adapted to a particular cellular compartment and/or set of metabolites. Endosymbiosis can also give rise to mosaic metabolic pathways comprised of enzymes with different evolutionary origins $[18,21]$. Proteins may be derived from the host, from the endosymbiont (both primary and secondary), or as a result of lateral gene transfer (LGT) from different prokaryotic and eukaryotic organisms. Understanding how cells adapt from living in a solitary state to having another organism within it is fundamental to understanding the evolution of plastid-bearing organisms.

We have sequenced the nuclear genome and transcriptome of the plastid-lacking goniomonad Go. avonlea CCMP3327 [7] with the goal of shedding light on its physiology and, more generally, the metabolic transformation that accompanied the transition from heterotrophy to phototrophy in its plastid-bearing sister taxa. Using comparative genomics and phylogenomics, we found little evidence for a photosynthetic ancestry in Go. avonlea and show that the acquisition of a plastid in an ancestor of present-day Cryptophyceae resulted in extensive reshuffling of metabolic pathways. Annotation of carbohydrate-active enzymes (CAZymes) [22] including glycosyltransferases (GTs), glycoside hydrolases (GHs), polysaccharide lyases (PLs), and carbohydrate esterases (CEs) allows us to make several predictions about the lifestyle of Go. avonlea and other goniomonads, including the possibility that they feed on multiple organisms, including eukaryotic algae.

\section{Methods}

\section{Cell culture, nucleic acid preparation, and genome} sequencing

Goniomonas avonlea CCMP3327 was grown in ESM medium [23] supplemented with ATCC's 1525 Seawater 802 medium. One day prior to harvesting, a dose of Penicillin-Streptomycin-Neomycin antibiotic mixture (Thermo Fisher cat \#15640055) was administered in order to reduce the number of co-cultured bacteria. Cells were harvested in two steps. First, liquid culture was filtered through a $2-\mu \mathrm{m}$ pore-sized polycarbonate membrane disc in order to deplete bacterial cells; the remaining protist cells were re-suspended in artificial seawater and transferred to a falcon tube. Cells were pelleted by centrifugation at 3000 RCF for $8 \mathrm{~min}$. DNA was extracted using a PureLink ${ }^{\odot}$ Genomic DNA Kit (Thermo Fisher Scientific, cat\# K182001). For RNA preparation, cells were lysed and phase-separated using TRIzol ${ }^{\mathrm{TM}}$ reagent (Thermo Fisher Scientific, cat \#15596018), followed by the use of the RNeasy Mini Kit (QIAGEN, cat \#74104) for precipitation, washes, and elution. 
DNA and RNA samples were sent to Génome Québec and the Beijing Genomics Institute (BGI) for library preparation and sequencing on the Illumina HiSeq2000 platform. At Génome Québec, genomic data were generated from a short-insert library, while $2 \mathrm{~kb}$ and $6 \mathrm{~kb}$ mate pair libraries were sequenced at the BGI. A total of $209,988,904,12,244,898$, and 35,906,071 forward and reverse reads, up to $100 \mathrm{bp}$ in length, were generated for the short-insert, $2 \mathrm{~kb}$, and $6 \mathrm{~kb}$ mate pair libraries, respectively. For the transcriptome, 62,428,409 forward and reverse reads were sequenced, up to $100 \mathrm{bp}$ in length, from a library prepared with the TruSeq protocol at Génome Québec.

\section{Genome assembly, gene prediction, and quality control}

Transcriptome reads were quality trimmed using Trimmomatic [24] and assembled de novo with Trinity [25]. The genome was assembled with ALLPATH-LG [26], Abyss [27], Minimus2 [28] and Ray [29]. We considered the N50 values of the two "best" assemblies (Abyss and ALLPATHS-LG) and used BOWTIE2 [30] coupled with ALE [31] and CGAL [32] to evaluate which assembly was optimal for our purposes. The ALLPATHS-LG assembly was selected and subjected to a blastn analysis [33]; contigs with bacterial hits with E-values lower than $1 \mathrm{e}^{-50}$ were considered bacterial and removed. We then predicted protein-coding genes using both Augustus [34, 35] and PASA [36], which allowed correction of gene models using transcriptome data. To further reduce the chance of bacterial contamination, we carried out blastp searches of our predicted proteins against NCBI $\mathrm{nr}$ (ftp:// ftp.ncbi.nlm.nih.gov/blast/db/) and the Marine Microbial Eukaryote Transcriptome Sequencing Project database (ftp://ftp.imicrobe.us/camera/) [37]. For each protein sequence, we took the first 10 hits and considered the protein to be from Eukaryota if $>60 \%$ of the hits were eukaryotic, or bacterial or archaeal if $>60 \%$ of the hits were to Bacteria or Archaea. Sequences that did not pass either threshold were assessed manually. In such cases, genes were mapped to their contigs and if $>60 \%$ of the gene models on the contig were eukaryotic, we considered the contig to be derived from the Goniomonas avonlea nuclear genome.

To identify as many protein-coding genes as possible and to ensure their full length, we predicted all ORFs from the Go. avonlea transcriptome, generating six frame translations for each transcript. From the pool of possible ORFs, we took the four longest translations and blasted them against the nr and MMETSP databases. All translated transcripts with a hit below $1 \mathrm{e}^{-05}$ were kept. To these transcriptome-derived sequences, we added protein sequences predicted from the genomic data using Augustus and PASA. The added sequences were those that had a hit $<1 \mathrm{e}^{-05}$ against the $\mathrm{nr}$ or MMETSP databases and that did not already match proteins from the transcriptome dataset with sequence identity $>90 \%$. The resulting set of 18,429 protein coding sequences, used for all subsequent analyses, represents the union of predicted gene models and predicted ORFs and represents a refined set of protein sequences demonstrably from Goniomonas and likely to have homologs in other organisms.

We assessed genome "completeness" using BUSCO (v1; [38]), which is based on a set of 429 protein-coding genes purported to be universally present in eukaryotes as single copies [37, 38]. The 18,429 Go. avonlea proteins were analyzed; the BUSCO results were compared to those obtained for $G u$. theta and the amoebozoan Dictyostelium discoideum.

\section{Orthologous protein annotation and KOG classification}

For Go. avonlea, Gu. theta, Bigelowiella natans, Emiliania huxleyi, Adineta vaga, and Arabidopsis thaliana, we clustered orthologous sequences using OrthoVenn (http:// www.bioinfogenome.net/OrthoVenn/) [39], with E-value and inflation value settings at $1 \mathrm{e}^{-5}$ and 1.5 , respectively. In addition, we compared the size and diversity of KOG functional categories (EuKaryotic Orthologous Groups) inferred from both the Go. avonlea and Gu. theta genomes using the WebMGA server (http://weizhonglab.ucsd.edu/webMGA/server/kog/) with an E-value cut-off of $1 \mathrm{e}^{-03}$ [40].

Protein annotation and sub-cellular localization prediction Protein annotation was performed using KOBAS [41]; proteins that were not annotated using this approach were analyzed using GhostKOALA [42]. Annotations were then used to generate KEGG metabolic pathway maps (http:// www.genome.jp/kegg/tool/map_pathway.html) [43].

In order to predict the sub-cellular locations of Go. avonlea proteins, we first selected 13,508 sequences inferred from our genome assembly that (i) start with a methionine and (ii) match the amino (N)-termini of proteins in our final set of 18,429 proteins, reasoning that proteins derived from our genomic (and not transcriptomic) data were more likely to possess full length $\mathrm{N}$ termini. Given the uncertainty of whether or not Go. avonlea and other goniomonads evolved from a plastid-bearing ancestor, we carried out different predictions using a combination of PredSL [44], TargetP [45], and Predotar [46] under the "plant" and "non-plant" modes (Additional file 1). Considering the formal possibility that Go. avonlea could, like Gu. theta, have a plastid acquired by secondary endosymbiosis, we used SignalP $4.1[47,48]$ coupled with ASAFind [49] to predict periplastidial compartment (PPC) and plastid proteins. For $\mathrm{Gu}$. theta, the predicted protein coding gene set from Curtis et al. [18] was used, as were the signal 
peptide predictions for the purposes of comparison with Go. avonlea.

\section{Annotation of carbohydrate-active enzymes (CAZymes)}

We performed a manual annotation of CAZymes [22] using a mix of BLAST [33] and HMM searches [50], similar to that done previously for $\mathrm{Gu}$. theta [18]. To assess the similarity between the two species across CAZyme families, we generated heat maps derived from an average linkage hierarchical clustering based on Bray-Curtis dissimilarity matrix distances and Ward's method [51-54]. The phylogenetic heat maps were generated with Rstudio software (https://www.rstudio.com/) using vegan in the R package (http://cc.oulu.fi/ jarioksa/ softhelp/vegan/html/vegdist.html) [55] with vegdist and hclust commands.

\section{Phylogenetic analysis of "algal" genes}

We sought to identify putative algal-derived homologs in the Go. avonlea genome by comparing its gene/protein set to that of Gu. theta and other algae. More specifically, we used blastp to search a custom database of 508 proteins predicted to be the product of endosymbiotic gene transfer (EGT) in Gu. theta [18]. For each Go. avonlea protein with a significant hit to this database (E-value $<1 \mathrm{e}^{-10}$ ), we used DIAMOND with the "more sensitive" option [56] to retrieve the top 2000 homologs above an E-value cut-off of $1 \mathrm{e}^{-10}$ from the $\mathrm{nr}$ and MMETSP [37] databases. Paralogs in the Go. avonlea candidate-EGT set were then identified by pairwise comparison of DIAMOND outputs; if two queries had $>50 \%$ overlap in hits they were considered paralogous and merged. Candidate Go. avonlea EGTs were annotated using InterPro [57] and their subcellular localizations were predicted as above.

Single-gene/protein trees were generated from alignments initially produced using MAFFT (version 7.205 [58]). Ambiguously aligned regions were removed using BMGE (version 1.1 [59]) with the BLOSUM30 scoring matrix and a block size of 4; trimmed alignments shorter than 50 amino acids were discarded. For the remaining candidates, an approximately maximum likelihood phylogeny was generated using FastTree [60] and used in an in-house tree-trimming script to reduce taxonomic redundancy in each dataset. Reduced datasets were then re-aligned using MAFFT-linsi (version 7.205 [58]), trimmed as above, and filtered to discard alignments shorter than 70 amino acids. Maximum-likelihood (ML) phylogenies were inferred for each remaining candidate in IQ-TREE (Version 1.5.5 [61]) under the LG4X substitution model [62] with 1000 ultra-fast bootstrap approximations (UFboot) [63]. The resulting trees were manually evaluated and sorted based on the topology of the Go. avonlea and $\mathrm{Gu}$. theta proteins in relation to each other and to sequences from various combinations of primary and secondary plastid-bearing photosynthetic lineages.

Additional genes/proteins of particular interest (for example, the CAZymes glycosyltransferase 28 and glucan water dikinase) were investigated on a case-by-case basis using a similar approach as above and as described in several other studies $[64,65]$. In these cases, homologs to predicted Go. avonlea genes/proteins were identified in various additional genomic/transcriptomic datasets, sequence redundancy was reduced using a combination of manual inspection and an automated treetrimmer analysis [66] and "final" alignments were produced with MUSCLE [67].

\section{Phylogenomics}

To investigate the phylogenetic position of Cryptista on the eukaryotic tree of life, a 250-marker gene, un-aligned dataset consisting of 150 operational taxonomic units (OTUs) corresponding to Burki et al. [14] was obtained from the Dryad Digital Repository [68]. The number of OTUs was systematically reduced to decrease the complexity of phylogenetic analyses while maintaining taxonomic diversity and minimizing missing data. Proteins predicted from the Go. avonlea transcriptome data were added to the dataset to increase marker gene coverage for the Goniomonadea. Go. avonlea homologs were identified using blastp with any Cryptista sequence (if available) or the first sequence in the marker gene set as the query; the best hit $\left(\mathrm{E}\right.$-value $\left.<1 \mathrm{e}^{-10}\right)$ in Go. avonlea was added to the dataset. Each marker gene/protein was aligned using MAFFT-linsi (version 7.205; [58]), and ambiguously aligned sites were removed using BMGE (version 1.1 [59]). Single gene trees were inferred using ML methods in IQTREE (Version 1.4.3 [61]) under the substitution model LG4X [62] with 1000 UFboot [63] and manually inspected to identify any obvious potential artifacts (e.g., long branch attractions). Marker genes/proteins were then realigned and subject to block removal prior to concatenation. The resulting supermatrix was used to infer a ML phylogeny in IQTREE (Version 1.4.3 [61]) using the model LG + C60 + F + PMSF [69] (selected according to the Bayesian Information Criterion (BIC) based on the outcome of a model test implemented in IQTREE [70]) with 100 standard bootstrap iterations.

In order to further investigate the phylogenetic position of Cryptista, phylogenetic trees were inferred (as above) based on modified versions of the supermatrix in which (i) sequences from plastid-bearing cryptistan taxa (i.e., Cryptophyceae) were removed from the dataset and (ii) PhyloMCOA [71] was used to identify and remove discordant genes in each OTU based on phylogenetic positioning across single-gene trees (using nodal distances) and multiple co-inertia analysis (MCOA). To evaluate the significance of differences in branch 
support, the standard error of bootstrap values was used with a 95\% confidence interval. To explore alternative signals emerging from Cryptista in the marker gene dataset, 183 of the 250 genes (those that contained a homolog in Go. avonlea and at least one other cryptomonad) were randomly partitioned into four equally sized bins. Each bin of marker genes was concatenated and used to infer a phylogeny based on ML methods in IQTREE (Version 1.4.3; [61]) under the model LG + $\mathrm{C} 20+\mathrm{F}$. This process was repeated 25 times, resulting in 100 randomly generated marker gene subset trees; for each tree, the phylogenetic position of Cryptista was manually evaluated.

\section{Results and discussion}

\section{The Goniomonas avonlea nuclear and mitochondrial genomes}

We sequenced the Go. avonlea nuclear genome to a depth of $\sim 24 \times$ coverage. In part due to the presence of repetitive sequences, the assembly is highly fragmented. For the final assembly, we retained contigs at least 500 bps in length resulting in 31,852 contigs (N50= 3831) totalling $91.5 \mathrm{Mb}$ and a GC content of $55.2 \%$ (Table 1). From our initial assembly, 33,470 genes were predicted; further investigation revealed that this number was artificially inflated due to assembly fragmentation. We thus merged protein-coding genes predicted from the genome with those inferred from transcriptome data (see "Methods"). This resulted in a set of 18,429 non-redundant protein-coding genes. When analyzed with BUSCO [38], our protein sequence data set was predicted to be $69 \%$ "complete," $20 \%$ "fragmented," and 9.7\% "missing"; this is comparable to the previously sequenced genome of $\mathrm{Gu}$. theta, which was inferred to be
78\% "complete," 12\% "fragmented," and 8.8\% "missing" (Additional file 2: Table S1). This suggests that despite the level of assembly fragmentation, the Go. avonlea protein coding gene set is similar in terms of completeness to that of $\mathrm{Gu}$. theta; conclusions about the presence/absence of metabolic pathways in the two genomes (see below) are probably not adversely affected by missing data. It should also be noted that in general such analyses are limited by a distinct lack of knowledge of cryptomonads and their large phylogenetic distance from the organisms used to create the BUSCO reference dataset. For reference, the well-annotated genome of the amoebozoan protist $D$. discoideum is inferred to be $5.1 \%$ "missing" using BUSCO (Additional file 2: Table S1).

Analysis of orthologous groups of proteins shared between the cryptomonads Go. avonlea and Gu. theta, as well as the rhizarian Bigelowiella natans, the haptophyte Emiliania huxleyi, the rotifer Adineta vaga, and the model land plant Arabidopsis thaliana, shows that, as expected, Go. avonlea and Gu. theta share more orthologous protein families with each other (4321 in total) than they do with other organisms (Go. avonlea shares 3647, 3441, 3173, and 2955 protein families with $B$. natans, E. huxleyi, A. vaga, and A. thaliana, respectively; Fig. 1a). Nevertheless, comparison of KOGs present in both Go. avonlea and Gu. theta reveals differences in the size and complexity of certain KOG functional categories (Fig. 1b). For example, KOG categories corresponding to cytoskeleton and intracellular trafficking, secretion, and vesicular transport are more abundant in Go. avonlea. Such differences may in part be due to the obligate phagotrophic lifestyle of Go. avonlea (see below). In contrast, Gu. theta appears somewhat enriched (relative to Go. avonlea) in functions associated

Table 1 General genome features for Guillardia theta and Goniomonas avonlea

\begin{tabular}{lll}
\hline & Guillardia theta & Goniomonas avonlea \\
\hline Assembly size & $87.1 \mathrm{Mb}$ & $91.5 \mathrm{Mb}$ \\
\# scaffolds & 669 & 31,852 \\
\# contigs & 5126 & 31,852 \\
N50 scaffolds & $40,445 \mathrm{bp}$ & $3831 \mathrm{bp}$ \\
GC\% & 52.9 & 55.2 \\
\# of protein coding genes & 24,822 & 33,470 \\
\# of introns & 132,885 & 112,740 \\
Percentage of genes with introns & $79 \%$ & $84 \%$ \\
Number of forward genes & 12,482 & 16,836 \\
Number of reverse genes & 12,441 & 16,638 \\
Average size of gene (nt) & 1863 & 1626 \\
Intron size average (nt) & 106 & 171 \\
Intron size mode (percent of total) & $47(5.1 \%)$ & 46 (4.7\%) \\
Average \# of introns per gene & 5.3 & 3.5 \\
\hline
\end{tabular}




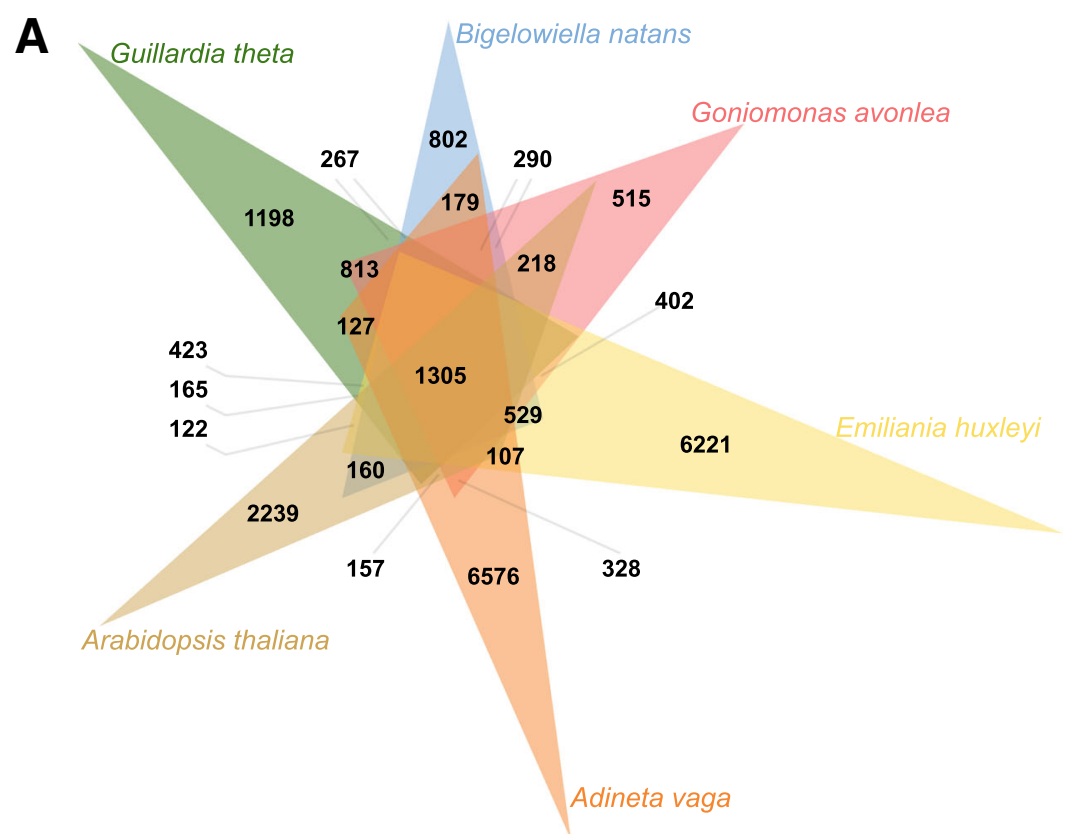

B

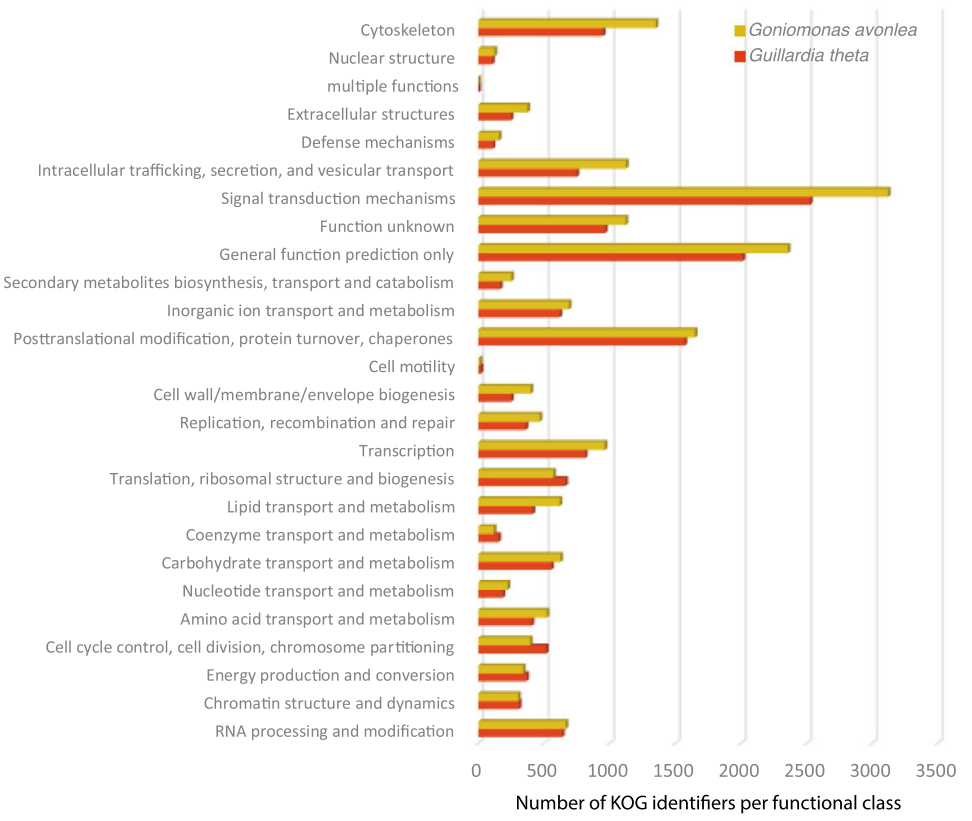

Fig. 1 Comparative genomics of Goniomonadea, Cryptophyceae, and other eukaryotes. a Venn diagram showing orthologous clusters shared between the goniomonad Goniomonas avonlea (red), the cryptophyte Guillardia theta (green), the rhizarian Bigelowiella natans (blue), the haptophyte Emiliania huxleyi (yellow), the opisthokont Adineta vaga (orange), and the land plant Arabidopsis thaliana (brown). Go. avonlea shares 4321 families with Gu. theta, higher than is shared with other eukaryotes (B. natans (3647), E. huxleyi (3441), A. vaga (3173), A. thaliana (2955)). b KOG classification of proteins in Go. avonlea (brown) and Gu. theta (red). Within most functional categories, the number of proteins in the two organisms is similar. However, Go. avonlea possesses more proteins in some categories, in particular the cytoskeleton and the intracellular trafficking, secretion, and vesicular transport families

with translation, ribosomal structure and biogenesis, as well as cell cycle control/division and chromosome partitioning (Fig. 1b).

We also sequenced and assembled the mitochondrial genome of Go. avonlea, which at $41.2 \mathrm{~kb}$ in size is similar to the circular mapping genomes of cryptophytes (when repeated sequences are excluded; e.g., [72, 73]) and the linear mtDNA of Palpitomonas bilix [74]. There are also interesting similarities amongst these mtDNAs in terms of gene content (Additional file 2: Figure S1). 
When compared to the mitochondrial genomes of other eukaryotic lineages, all members of Cryptista considered here share very similar gene repertoires, especially for complexes I-V of the electron transport chain (Additional file 2: Figure S1): they all have nad1-4, $4 L$, 5-11, sdh3, 4, cob, cox1-3, atp1, 3, 4, 6, 8, and 9. That said, Go. avonlea and P. bilix share an rpl2 gene that is not present in cryptophyte mtDNAs, and all sequenced cryptophyte mtDNAs possess an rps2 gene [73] that is not present in Go. avonlea or P. bilix. Go. avonlea mtDNA also lacks the tatA and tatC genes found in other Cryptista mtDNAs, which encode components of the twin arginine translocator. Of particular note, like cryptophytes, Go. avonlea lacks the mitochondrial $c c m A$, $B, C$, and $F$ genes recently found in the mtDNA of $P$. bilix [74]. These genes encode a bacterial-type cytochrome $c$ maturation system ("system I"); our data support the hypothesis that goniomonads use a nucleus-encoded holocytochrome c synthase (HCCS) system (i.e., "system III"). We searched for, and found, the HCCS gene in the nuclear genome of Go. avonlea (comp53045_c0_seq2_6_ORF10_179 and comp39203_c0 _seq2_6_ORF3_158). This confirms the authenticity of such genes found in transcriptome data from Go. pacifica and the katablepharid Roombia sp. NY0200 [74]. With its mitochondrial-encoded "system I" cytochrome $c$ maturation system, $P$. bilix is thus an outlier amongst cryptistan protists, which raises interesting questions about how the type I and III systems evolved in these and other organisms (see Nishimura et al. [74] for discussion). All things considered, our mitochondrial genome analyses are consistent with phylogenomic data suggesting that although the organisms that comprise Cryptista are not closely related, they represent a monophyletic assemblage on the eukaryotic tree of life $[9,10,14]$.

\section{Goniomonas avonlea does not have a plastid}

On the basis of electron microscopy, Go. avonlea cells do not have any obvious plastid-like internal structures [7]. Nevertheless, with complete genome and transcriptome sequences in hand, we explored its predicted metabolic pathways as well as putative TOC-TIC proteins in an effort to detect any hint of evidence for a cryptic plastid-none was found (Additional file 2: Figure S2). Moreover, we predicted the sub-cellular locations of all of the Go. avonlea proteins under the following hypothetical scenarios: (i) the organism does not have a plastid, (ii) it has a cryptic plastid derived from primary endosymbiosis, or (iii) it has a cryptic plastid of secondary endosymbiotic origin. In short, we found no evidence supporting the presence of a plastid of primary or secondary endosymbiotic ancestry; hundreds of candidate proteins were identified using various search procedures (e.g., presence of bipartite $\mathrm{N}$-terminal targeting sequences) but closer investigation revealed these to be false positives (Additional file 1 and Additional file 2: Figure S3). This is consistent with previous analyses performed on transcriptome data from Goniomonas pacifica [10] as well as microscopic observations of several Goniomonas strains, including Go. avonlea [7, 75, 76].

\section{Absence of endosymbiotically derived algal genes in Goniomonas avonlea}

Curtis et al. [18] identified 508 genes of probable endosymbiont (i.e., algal) ancestry in the Gu. theta nuclear genome. Many of these endosymbiotic gene transfers (EGTs) encode proteins that are predicted to have been repurposed and to function in the host cytosol of $G u$. theta or other host-associated compartments; if Go. avonlea lost a red-algal-derived plastid secondarily, one might thus predict that at least some of these algal genes would still be present in its genome [18, 77]. Using sequence homology searches, we found that Go. avonlea has one or more homologs to 212 of the $508 \mathrm{Gu}$. theta EGT genes (285 Go. avonlea proteins in total). Manual investigation of the phylogenies of each of these 285 proteins (Fig. 2) revealed that only six show any obvious red algal signal in both Cryptophyceae (including $G u$. theta) and Go. avonlea, none of which were predicted to be targeted to a plastid or function in plastid metabolism. In contrast, the Cryptophyceae showed a significant red-algal signal to the exclusion of Go. avonlea in 75 of these 285 phylogenies (e.g., tryptophanyl-tRNA synthetase; Fig. 3). Similar to the results of Curtis et al. [18], a large proportion of these trees were found to be ambiguous with respect to the nature of their algal signal. In some cases, the cryptophyte homologs branch closest to green or glaucophyte algae (31/285 and $97 / 285$ trees where a Go. avonlea homolog branches with or without the predicted $G u$. theta EGT in the phylogeny, respectively), while in others the primary algal lineage is entirely unclear (12/285 trees without a Go. avonlea homolog branching with the predicted cryptophyte EGT, 13/285 where a Go. avonlea homolog branches with the predicted cryptophyte EGT). However, given that the phylogenetic position of Cryptista relative to Archaeplastida and other eukaryotic supergroups is unclear [14], extreme caution is needed when considering these "green," "glaucophyte" or ambiguous algal genes as bona fide EGTs, particularly in cases where obvious plastid targeting signals and/or plastid-associated functions are not observed [18]. Here, plastid-targeting signals and/or plastid-associated functions were not observed for any Go. avonlea homolog that branched with a Gu. theta predicted EGT showing a common "green," "glaucophyte," or ambiguous algal phylogenetic signal.

Is this small "red algal" footprint in the Go. avonlea genome (6/508 predicted algal EGTs in Gu. theta) 


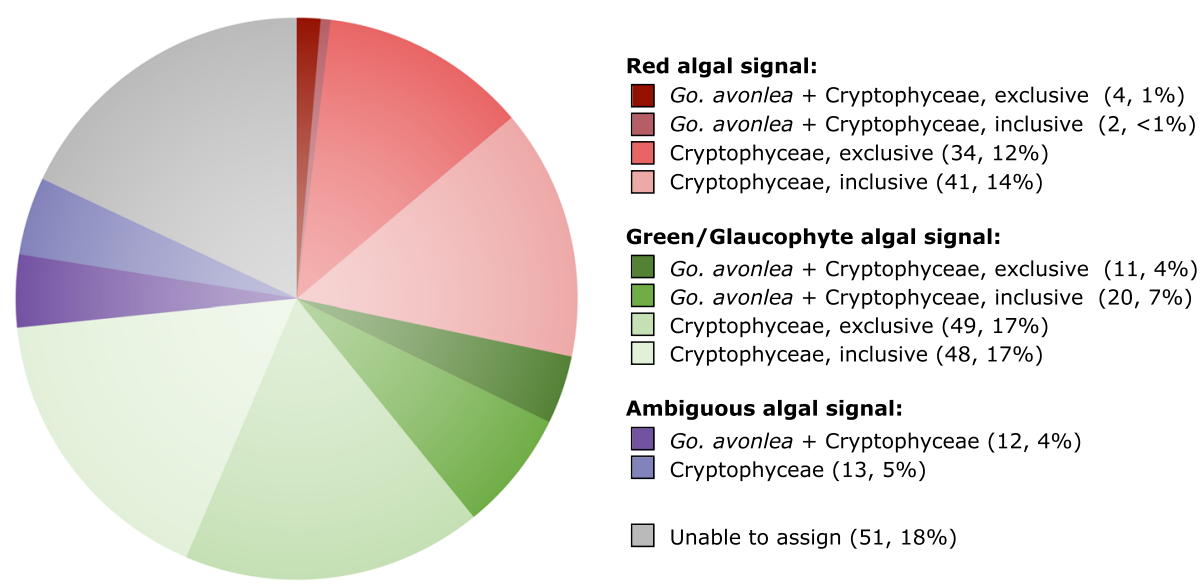

Fig. 2 Algal genes in Guillardia theta and Goniomonas avonlea. The diagram shows the distribution of topologies observed in Go. avonlea homologs to 508 "algal" endosymbiotic gene transfers predicted by Curtis et al. [18] in Gu. theta. Phylogenies were evaluated and sorted based on the relative positioning of Go. avonlea and Gu. theta (and other Cryptophyceae) and their relationship to Archaeplastida lineages and secondarily photosynthetic taxa. An exclusive relationship indicates a direct relationship with an Archaeplastida lineage while an inclusive one indicates there are intervening secondarily photosynthetic taxa. A given topological pattern was only assigned if the corresponding UFboot support was greater than $80 \%$. Of the 285 homologs identified in Go. avonlea, only six show an affinity to Cryptophyceae and red algae

meaningful? Comparing this signal to that observed against a control taxon (i.e., an unrelated amoebozoan with an unambiguous non-photosynthetic ancestry) allowed us to assess the expected signal due to background phylogenetic noise [77]. We found that Go. avonlea appeared sister to Amoebozoa in 24/285 single-gene trees, substantially higher than the red algal fraction. These analyses strongly suggest that the common red-algal footprint in Go. avonlea and Gu. theta is not significant, consistent with the lack of evidence for the existence of a cryptic plastid from microscopy and protein subcellular localization predictions. It is unclear to what extent our phylogenomic results are biased by taxonomic sampling; genome sequence data are presently stacked in favor of green algae and land plants over red algae [15]. It will thus be interesting to see whether the number of "red-algal" genes in Cryptophyceae (as well as other complex red algae-derived plastid bearing taxa) and plastid-lacking lineages such as Go. avonlea will go up or down as databases become more inclusive.

Considering the Go. avonlea predicted proteome as a whole, a top blast hit analysis revealed an expected affinity to other Cryptista $\sim 33 \%$ of the time, with the next most common top hits being to Alveolata $(\sim 13 \%)$, Viridiplantae $(\sim 12 \%)$, and Stramenopiles ( 11\%) (Fig. 4). Notably, the number of instances in which an amoebozoan protein was the most similar sequence (1263 proteins, $8 \%$ ) was considerably greater than those where a red algal homolog was most similar (128, 0.7\%), suggesting again that the red algal signal in the Go. avonlea genome is minimal and not the result of EGT. In the case of the phototroph $\mathrm{Gu}$. theta, a $\sim 4.4$ times enrichment in red algal signal relative to Go. avonlea was seen in a top blast hit analysis and a $\sim 4.5$ times greater enrichment in terms of archaeplastidal signal (compared to an amoebozoan control and adjusted for relative database representation to minimize database composition bias [77]). There is thus no indication of a red algal signal above background noise in the Go. avonlea genome on the basis of top blast hits.

\section{The position of Cryptista on the eukaryotic tree of life}

The Cryptista comprises a diverse collection of heterotrophic and photosynthetic lineages, one that has only recently been recognized as a monophyletic entity $[10,78]$. Not surprisingly, Cryptista has been difficult to place in the eukaryotic tree of life; some or all of its members have been shown to branch sister to Haptophyta (e.g., [8]) or, alternatively, sister to Archaeplastida (e.g., [13]). Phylogenies inferred here based on a modified Burki et al. [68] dataset (98 OTUs, 250 marker genes) recovered identical relationships to those inferred by Burki et al. [14]; however, we were able to evaluate branch support using standard bootstrapping under the complex model LG + C60 + F + PMSF (Fig. 5) [69]. In Burki et al. [14], Bayesian analyses on the original dataset did not result in global convergence, an issue that is common when analyzing such large phylogenomic datasets (e.g., [13]). Nevertheless, these authors considered the tree topology resulting from the non-converged Bayesian analysis and found only minor differences with regard to the position of the Cryptista to Archaeplastida. Due to the large size of our dataset, and in light of the observations of Burki et al. [14], we did not attempt Bayesian analyses; we instead focused on the ML analysis whose tree topology could be statistically evaluated 


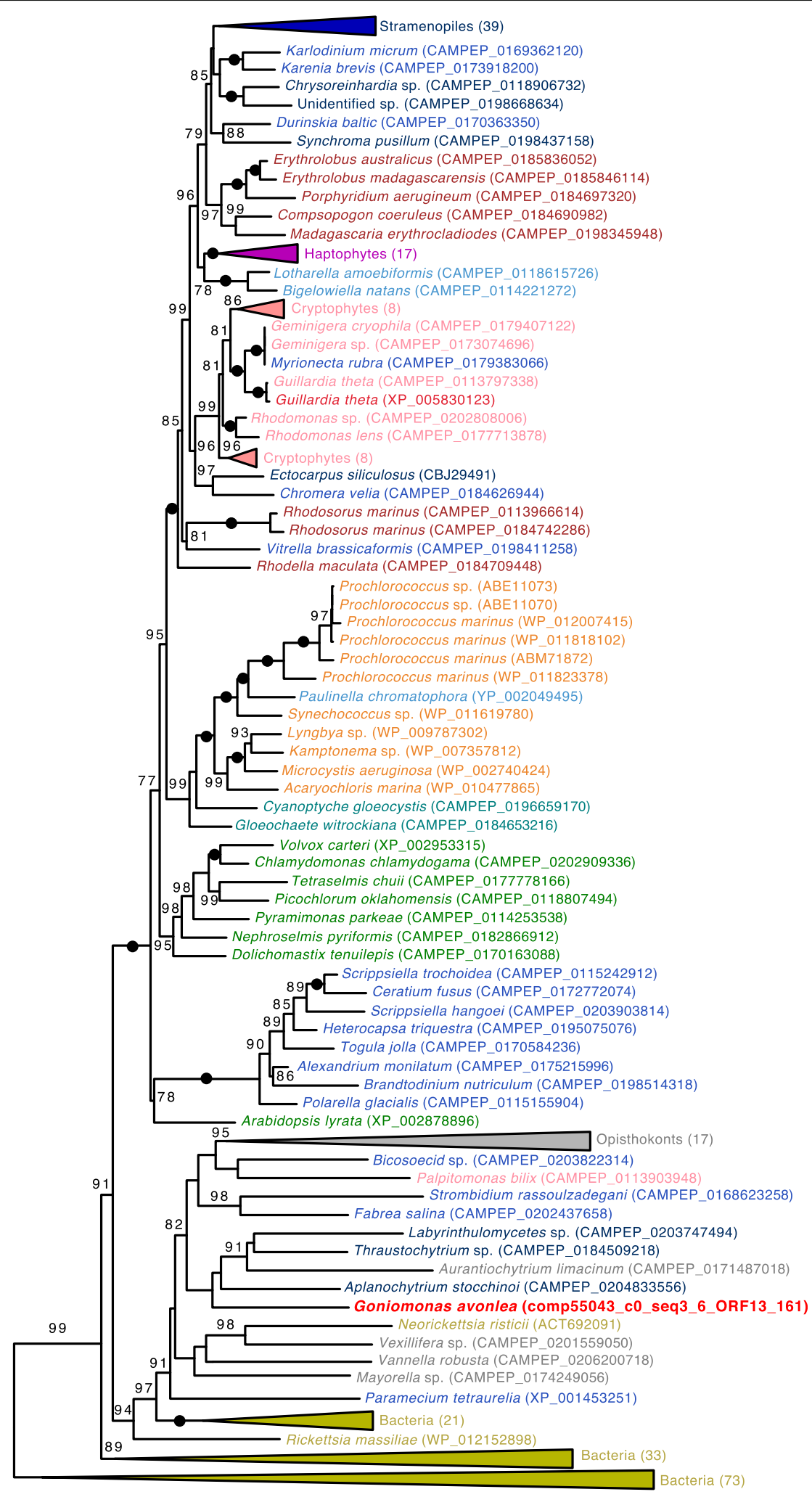

0.3

Fig. 3 (See legend on next page.) 
(See figure on previous page.)

Fig. 3 Maximum likelihood (ML) phylogeny of tryptophanyl-tRNA synthetase in diverse eukaryotes and prokaryotes. The tree was inferred under the model LG4X (with 100 standard bootstrap replicates) and shows an apparent red algal ancestry for homologs in Cryptophyceae but not in Go. avonlea. Eukaryotic OTUs are colored according to their known or predicted "supergroup" affinities with sequences from Go. avonlea and predicted Gu. theta EGTs [17] highlighted in bright red (Viridiplantae are in green, Glaucophyta are in turquoise, Rhodophyta are in dark red, Cyanobacteria are orange and other Bacteria are in gold, Cryptophyta are in pink, Haptophyta are in purple, Stramenopiles are in dark blue, Alveolata are in blue, Rhizaria are in light blue). The tree shown is midpoint rooted. Black dots indicate maximal support for particular nodes. When not maximal, only bootstrap support values $>70 \%$ are shown. The scale bar shows the inferred number of amino acid substitutions per site

using standard (i.e., nonparametric) bootstrapping. With the exception of Archaeplastida, and the Excavata (whose monophyly is still debated; e.g., see [79]), the monophyly of eukaryotic supergroups (including SAR) was recovered with maximum support, and Haptista (i.e., haptophytes + centrohelids) branched with nearly maximum support as sister to SAR (99\% standard bootstrap support). The monophyly of Archaeplastida was disrupted by the positioning of Cryptista, which was found to branch with Archaeplastida with maximum support; more specifically, Cryptista branched with a standard bootstrap value of $82 \%$ as sister to Viridiplantae and Glaucophyta (99\% standard bootstrap support) to the exclusion of Rhodophyta.

Removing single genes in specific OTUs determined to be discordant via PhyloMCOA [71] did not change the tree topology, but rather significantly increased the support of Cryptista branching internal to Archaeplastida (90\% standard bootstrap support), suggesting that the observed relationship is not caused by a few genes in Archaeplastida and Cryptista that overwhelm the dataset with non-phylogenetic signal. The removal of Cryptophyceae from the dataset also resulted in no change in tree topology, recovering non-photosynthetic Cryptista as sister to Viridiplantae and Glaucophyta to the exclusion of Rhodophyta with $75 \%$ standard bootstrap support (not substantially different from Fig. 5), suggesting that this association is not entirely due to the presence of plastid-bearing lineages. It remains possible, however, that instances of EGT have gone undetected within Cryptista due to the close evolutionary relationship of their nuclear genes (either Viridiplantae and Glaucophyta specifically or Archaeplastida as a whole) and the source of their plastid (Rhodophyta), making it extremely difficult to disentangle the source of genes in the nucleus and resolve the exact position of the phylum Cryptista within eukaryotes [80].

Further investigation into the position of Cryptista on the eukaryotic tree of life using random subsets of marker genes resulted in Cryptista branching consistently with some combination of one or more Archaeplastida sub-groups (93/100 iterations) (Fig. 6). While Cryptista was most frequently observed as sister to the clade comprising Viridiplantae and Glaucophyta (30\%), it was also often recovered as sister to Glaucophyta exclusively (24\%), Rhodophyta exclusively (13\%), and to a monophyletic Archaeplastida (20\%). Interestingly, in stark contrast to the $24 \%$ of iterations that resulted in a Cryptista-Glaucophyta-specific relationship, only $3 \%$ showed Cryptista branching with Viridiplantae exclusively. This may suggest that Cryptista shares a closer ancestry with Glaucophyta, but it could also simply be the result of similar compositional biases or slow evolutionary rates causing "short branch exclusion" [81].

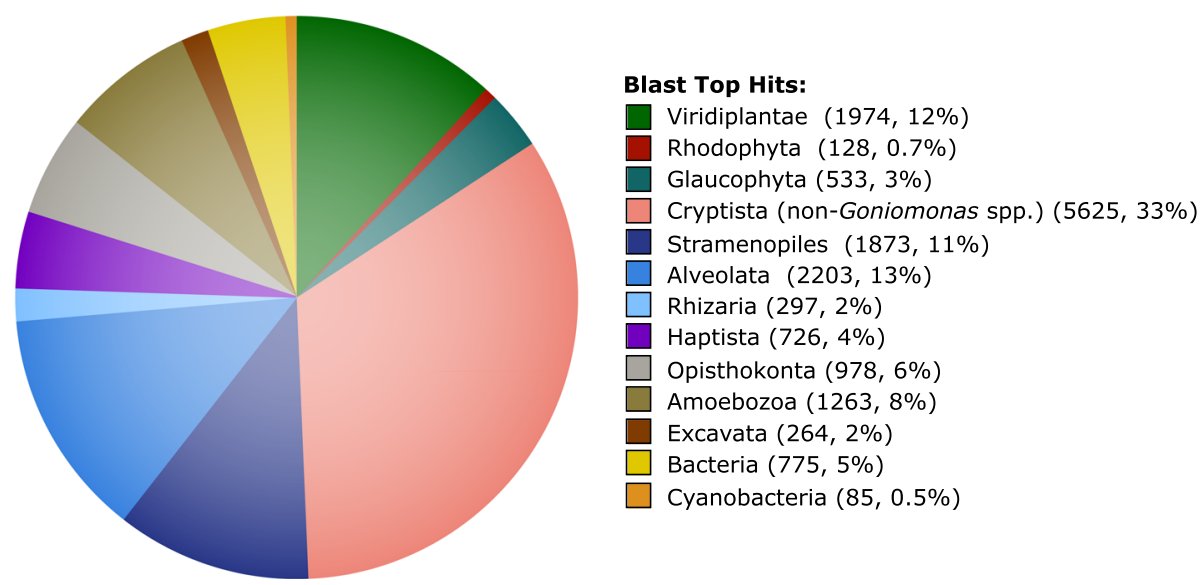

Fig. 4 Taxonomic distribution of top blast hits for Goniomonas avonlea proteins. The top blast hit was defined as the most significant homolog to Go. avonlea (i.e., lowest E-value with a cutoff of $1 \mathrm{e}^{-10}$ ) excluding any other Goniomonas sequence 


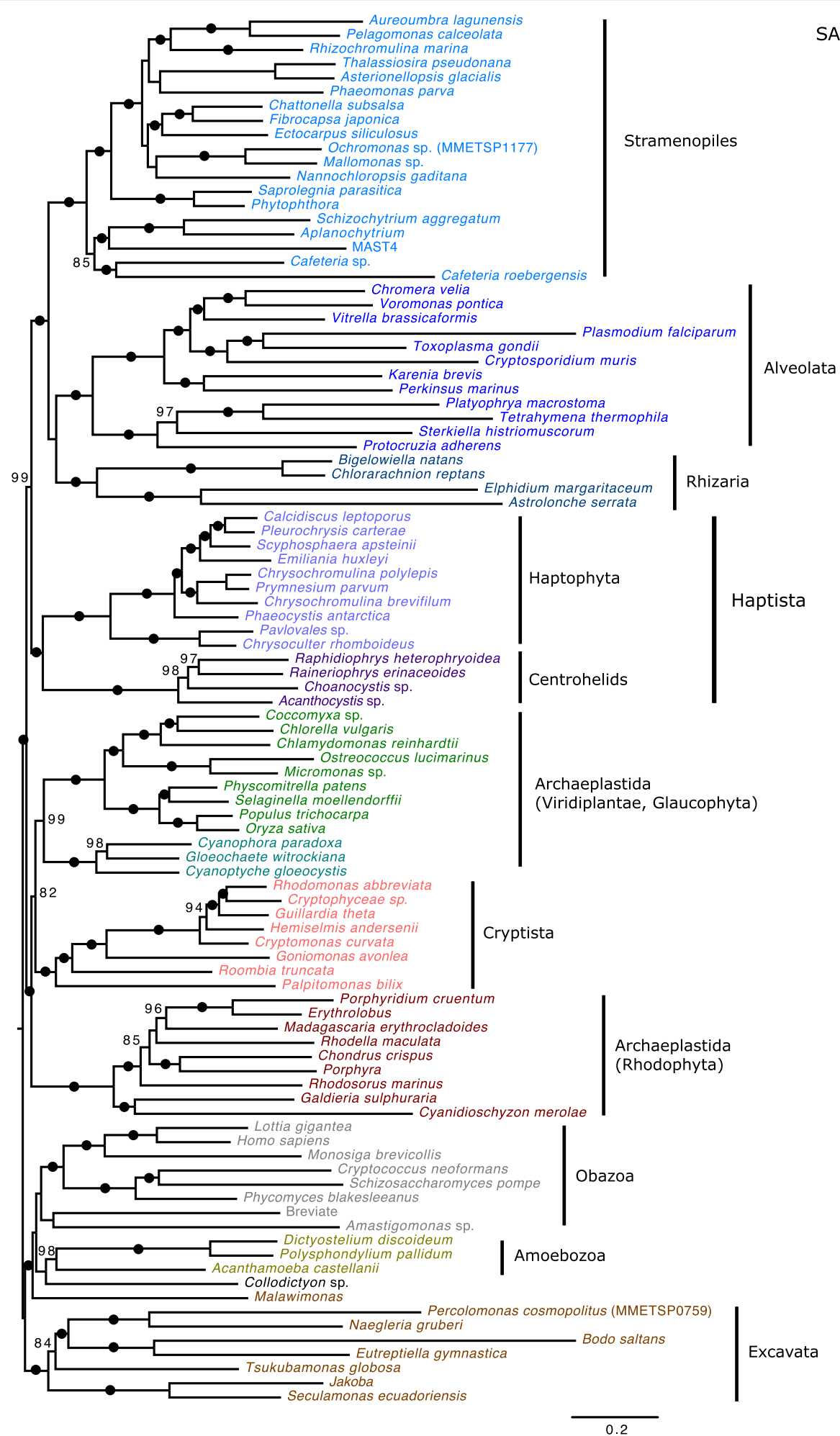

Fig. 5 Phylogenomic analysis of the eukaryotic tree of life. Tree shown is a maximum likelihood (ML) phylogeny of a 250 marker gene/protein dataset as in Burki et al. [14] that includes new transcriptome data from Go. avonlea. The phylogeny is based on a concatenated marker gene alignment of 71,151 unambiguously aligned sites across 98 OTUs inferred under the model LG + C60 +F + PMSF with 100 standard bootstrap replicates. The tree shown is midpoint rooted. Black dots indicate maximal support for a particular node. When not maximal, only bootstrap support values $>70 \%$ are shown. The scale bar shows an inferred 0.2 substitutions per site 


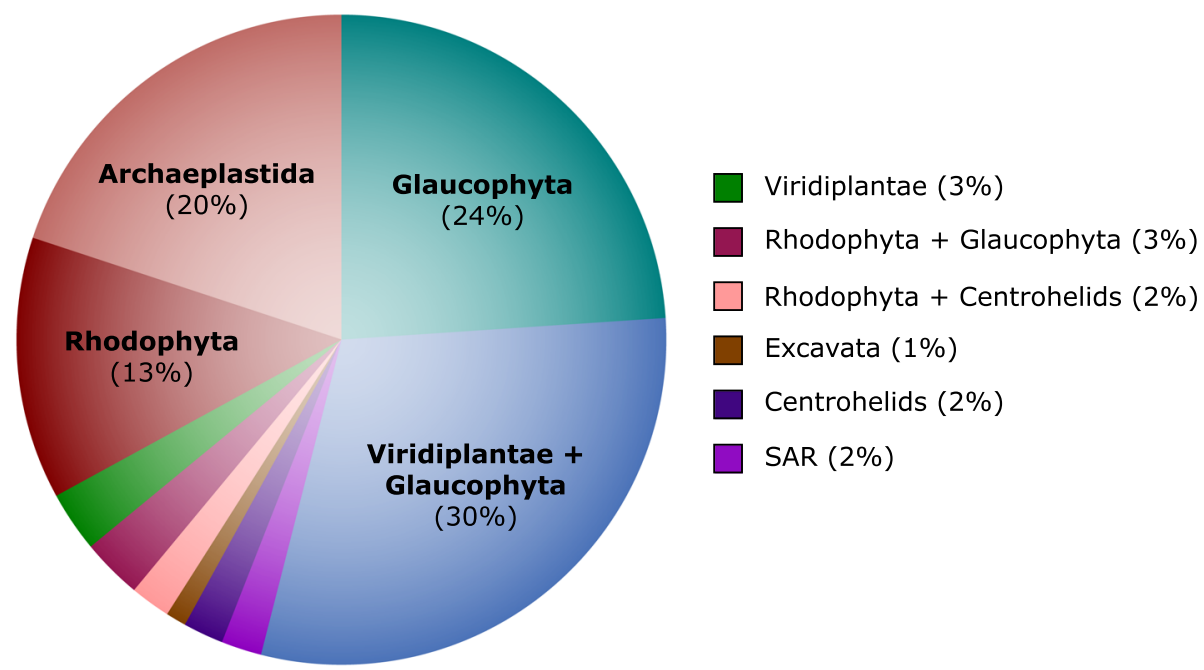

Fig. 6 Impact of gene sampling on the phylogenetic position of Cryptista on the tree of eukaryotes. The diagram shows the phylogenetic position of Cryptista within each ML tree inferred using randomly generated subsets of 250 marker genes from the Burki et al. [14] dataset (four gene bins were used; for each iteration, three bins had 46 genes and one bin had 47 genes). Only marker genes for which a homolog was present in Goniomonas avonlea and at least one additional Cryptista were included. The distribution shown is based on a total of 100 randomly generated marker gene subset trees

Notably, a sister relationship between Cryptista and Haptophyta/Haptista was never observed. While the exact position of Cryptista relative to Archaeplastida is uncertain, its association with Archaeplastida appears stable. As discussed above, this relationship makes it difficult to assign "algal genes" as EGTs in Cryptista, and it remains to be determined if they are of endosymbiotic origin or vertical ancestry.

\section{Metabolic "rewiring" in Guillardia theta linked to plastid acquisition}

Given that there is no plastid in Go. avonlea, we compared the predicted metabolic capacities of Gu. theta and Go. avonlea with the goal of deducing the metabolic and enzymatic functions gained with the acquisition of a red algal-derived secondary plastid. In our study, four main biochemical pathways/processes are predicted to be plastid-localized in Gu. theta and thus obviously related to plastid acquisition: photosynthesis, isoprenoid biosynthesis via the non-mevalonate (MEP/DOXP) pathway, carotenoid biosynthesis, and porphyrin and chlorophyll metabolism (Additional file 2: Figure S4-S8). Several other pathways that may also have been acquired by secondary endosymbiosis but are not obviously plastid-localized in Gu. theta are ubiquinone and terpenoid-quinone biosynthesis, as well as thiamine biosynthesis (Additional file 2: Figure S9 \& S10). As expected, $\mathrm{Gu}$. theta pathways clearly localized to the plastid include those associated with pigment biosynthesis and photosynthesis (carotenoid biosynthesis, chlorophyll and porphyrin biosynthesis (Additional file 2: Figure S7 \& S8)). The presence of a thiamine (vitamin B1) pathway (Additional file 2: Figure S10), which does not appear to be plastid-localized, as well as ubiquinone and menaquinone/ phylloquinone biosynthesis, which are involved in electron transport, also seems to correlate with secondary plastid acquisition in Cryptophyceae. It should be noted that while menaquinone biosynthesis should take place in the plastid, signal peptides have not been detected on the requisite proteins in $\mathrm{Gu}$. theta [18]. We also observed that while a peroxisome-localized primary bile biosynthesis pathway is present in Go. avonlea, it is apparently absent in Gu. theta (Additional file 2: Figure S11). This suggests either loss of this pathway in Gu. theta or later acquisition in Go. avonlea.

In Go. avonlea, fatty acid biosynthesis is predicted to occur partly in the mitochondrion (FabF and FabB) and partly in the cytosol (FAS1) (Additional file 2: Figure S4, S12, S13); while in Gu. theta, it is predicted to be plastid-localized. Interestingly, while the mevalonate pathway in Go. avonlea is found in the cytosol (Additional file 2: Figure S4, S6), Gu. theta possesses both the mevalonate and MEP/DOXP pathways, which use acetyl-CoA and GA3P (D-glyceraldehyde-3-phosphate) with pyruvate, respectively, to synthesize isoprenoid precursor (Additional file 2: Figure S2 and S6). Gu. theta (and perhaps other Cryptophyceae) thus appear to have redundant metabolic capacities with which to synthesize isopentenyl diphosphate (i.e., either the mevalonate or the MEP/DOXP pathway) which may represent the ancestral eukaryotic metabolism or the endosymbiotically derived one, respectively. 


\section{Storage polysaccharides in Goniomonas avonlea}

Alpha glucans are the most common storage polysaccharides and can be found in different forms (e.g., glycogen and starch). Production of alpha glucans can be assessed by the presence of certain CAZyme families: glycoside hydrolase (GH)13, glycosyltransferase (GT)35 and GT5 for all organisms, and GT3, GH133, and GT8 for eukaryotes in particular [82]. These enzymes are all found encoded in the Go. avonlea genome and are very similar to those involved in classical eukaryotic glycogen metabolism (Table 2). Several proteins with GT8 domains can putatively be assigned as glycogenins since their best blast hits are to bona fide glycogenins in other organisms such as Saccharomyces cerevisiae, albeit with poor E-values (data not shown). Additionally, a complete metabolic pathway for the production of an alpha glucan storage polysaccharide seems to be present in Go. avonlea, as supported by the presence of catabolic enzymes such as GH13 and GH14. Because of the presence of the carbohydrate-binding module 45 (CBM45) coupled to a pfam01326 domain (corresponding to a glucan water dikinase (GWD); see below) (Table 2), Go. avonlea could be a starch accumulating organism, even though electronic microscopy has not revealed the presence of starch granules [7].

In addition to genes associated with alpha glucan metabolism, the Go. avonlea genome encodes putative beta glucan-specific proteins, i.e., enzymes falling in the GT2 and GT48 families (Table 2). These enzymes are implicated in either the production of cellulose in the cell wall or the synthesis of beta storage polysaccharides [83]. No genes for GH9 enzymes were found in the Go. avonlea genome, consistent with the fact that cellulose has not been observed in goniomonads [76]. Even if we cannot exclude the possibility of the presence of glucan in the periplast component of cryptomonads, we suggest that the presence of GT2 and GT48 family enzymes could be related to the synthesis of beta storage polysaccharides. The catabolism of beta polysaccharides in Go. avonlea could be performed by GH16 family enzymes, laminarinase in particular. However, the laminarinase-like enzymes appear to be secreted, suggesting they are involved in the degradation of exogenous rather than endogenous polysaccharides (Table 2).

\section{Goniomonas avonlea appears capable of digesting both bacteria and eukaryotes}

Many heterotrophic eukaryotes ingest bacteria by phagocytosis and Go. avonlea is no exception. The CAZy database includes glycoside hydrolases (GHs) clustered into 136 families, and our analysis of carbohydrate-active enzymes (CAZymes) in Go. avonlea provides insight into what its prey might be. The Go. avonlea genome contains genes for three families of signal peptide-containing lysozymes (GH22, GH24, and GH25) (Table 2) that are likely associated with bacterial phagocytosis. The GH2 family in Go. avonlea also includes several enzymes with secretion signals (Table 2). Interestingly, the presence of several GH enzymes suggests that phagocytosis in Go. avonlea may also involve eukaryotic prey, specifically algae: these are proteins belonging to the GH45, GH5, and GH3 families, which are putative cellulases, agarases (GH50), and putative hemicellulases (GH43 and GH54; Table 2). Although the cellulases have signal peptides, suggesting that they are involved in the degradation of algal cellulose, it should be noted that cellulose is also found in some bacteria. More intriguing is the identification of genes for signal peptide-containing agarases (GH50) in the Go. avonlea nuclear genome, since agar is found only in red algae [84] (Table 2). This suggests that Go. avonlea could feed on red algae by phagocytosis, although agarase is also known to degrade alginate, which is found in some bacterial biofilms. The presence of putative secreted hemicellulases in Go. avonlea is also consistent with the hypothesis that Go. avonlea preys on algae. Several amylases (GH13) and two beta-amylases (GH14) were found to have signal peptides, and may therefore be involved in the degradation of storage polysaccharides from organisms taken up by phagocytosis (Table 2).

While plastid-bearing photosynthetic organisms fix carbon through the Calvin cycle and transform it into sugars for various purposes (most notably, energy), heterotrophic organisms need to acquire sugar from their environment. Thus, photosynthetic organisms typically possess fewer GHs than heterotrophic organisms. This general pattern holds when the heterotroph Go. avonlea is compared to the phototroph Gu. theta. Go. avonlea possesses $183 \mathrm{GHs}$ (111 of which are predicted to be secreted), compared to only 57 in Gu. theta (Table 2). Gu. theta also appears to lack certain $\mathrm{GH}$ families that are typically absent in autotrophs. Nevertheless, 18 of the 57 GHs in Gu. theta are predicted to be secreted, consistent with the gene-based model that predicts Gu. theta to be mixotrophic [85], as has been suggested for several other Cryptophyceae [86-88]. Another interesting observation is the co-occurrence of certain GH families in Gu. theta and Go. avonlea, most notably GH116. However, whereas Go. avonlea is predicted to secrete these enzymes in order to obtain exogenous polysaccharides, $G u$. theta presumably uses them to digest its own endogenous polysaccharides (Table 2). Moreover, while Gu. theta does not have more GTs than Go. avonlea, some classes that are only present in Gu. theta (GT14, GT29) are involved in protein glycosylation (Table 2).

\section{Global CAZome analysis}

In order to better understand the biology of Go. avonlea relative to $G u$. theta and vice versa, we performed a global 
Table 2 CAZy family enzymes in Goniomonas avonlea and Guillardia theta (those predicted to be secreted are in parentheses)

\begin{tabular}{|c|c|c|c|c|c|}
\hline & \\
\hline CAZy families & Go. avonlea & Gu. theta & CAZy families & Go. avonlea & Gu. theta \\
\hline $\mathrm{GH} 2$ & $5(4)$ & $4(0)$ & GH99 & $3(0)$ & $5(1)$ \\
\hline $\mathrm{GH} 3$ & $10(7)$ & 0 & $\mathrm{GH} 110$ & $1(1)$ & 0 \\
\hline GH5 & $12(8)$ & $4(1)$ & $\mathrm{GH} 113$ & $1(0)$ & 0 \\
\hline GH9 & 0 & $1(0)$ & GH115 & $1(0)$ & 0 \\
\hline GH13 & $13(3)$ & $5(4)$ & GH116 & $2(2)$ & $1(0)$ \\
\hline $\mathrm{GH} 14$ & $2(1)$ & $3(1)$ & $\mathrm{GH} 128$ & $2(1)$ & 0 \\
\hline GH15 & $1(1)$ & 0 & GH130 & $3(1)$ & $1(0)$ \\
\hline GH16 & $4(3)$ & 0 & GH133 & $2(0)$ & 0 \\
\hline $\mathrm{GH} 17$ & $1(1)$ & 0 & & & \\
\hline $\mathrm{GH} 18$ & $3(3)$ & 0 & CBM13 & $1(1)$ & $1(0)$ \\
\hline $\mathrm{GH} 20$ & $16(8)$ & $4(1)$ & CBM20 & $10(0)$ & $17(8)$ \\
\hline $\mathrm{GH} 22$ & $1(1)$ & 0 & CBM32 & $1(0)$ & $3(2)$ \\
\hline $\mathrm{GH} 24$ & $1(1)$ & 0 & CBM45 & $1(0)$ & 0 \\
\hline $\mathrm{GH} 25$ & $3(2)$ & 0 & CBM47 & $4(3)$ & $3(3)$ \\
\hline $\mathrm{GH} 27$ & $11(9)$ & $2(1)$ & CBM48 & $9(0)$ & $6(2)$ \\
\hline $\mathrm{GH} 28$ & $5(4)$ & 0 & & & \\
\hline $\mathrm{GH} 29$ & $5(4)$ & $2(1)$ & GT1 & $10(0)$ & $4(0)$ \\
\hline GH30 & $2(2)$ & 0 & GT2 & $19(0)$ & $22(0)$ \\
\hline GH31 & $7(4)$ & $3(1)$ & GT3 & $2(0)$ & 0 \\
\hline GH32 & $1(1)$ & 0 & GT4 & $18(0)$ & $27(0)$ \\
\hline GH33 & $3(2)$ & 0 & GT5 & $2(0)$ & $6(0)$ \\
\hline GH35 & $1(1)$ & $1(1)$ & GT6 & $3(0)$ & $3(0)$ \\
\hline GH36 & 0 & 7 (2) & GT7 & $1(0)$ & $2(1)$ \\
\hline $\mathrm{GH} 37$ & $1(0)$ & 0 & GT8 & $17(0)$ & $14(0)$ \\
\hline GH38 & $9(6)$ & $1(0)$ & GT10 & $11(0)$ & $8(0)$ \\
\hline GH39 & $3(3)$ & 0 & GT11 & $4(0)$ & $3(0)$ \\
\hline $\mathrm{GH} 43$ & 7 (7) & 0 & GT13 & $4(0)$ & $6(0)$ \\
\hline $\mathrm{GH} 45$ & $1(1)$ & 0 & GT14 & 0 & $2(0)$ \\
\hline GH47 & $10(3)$ & $5(1)$ & GT15 & $5(0)$ & $5(0)$ \\
\hline GH50 & $1(1)$ & 0 & GT16 & $1(0)$ & $3(0)$ \\
\hline GH51 & $1(0)$ & 0 & GT17 & $10(0)$ & $5(0)$ \\
\hline GH54 & $1(1)$ & 0 & GT18 & $1(0)$ & $1(0)$ \\
\hline GH55 & $2(1)$ & 0 & GT19 & $1(0)$ & $1(0)$ \\
\hline GH56 & $2(2)$ & 0 & GT20 & $4(0)$ & $4(0)$ \\
\hline GH63 & $4(0)$ & 0 & GT22 & $6(0)$ & $3(0)$ \\
\hline GH65 & $3(2)$ & 0 & GT23 & $16(0)$ & $16(0)$ \\
\hline GH76 & $1(0)$ & 0 & GT24 & 0 & $1(0)$ \\
\hline GH77 & $1(0)$ & $5(2)$ & GT25 & $1(0)$ & $1(0)$ \\
\hline GH78 & $6(3)$ & 0 & GT26 & $1(0)$ & 0 \\
\hline GH79 & $4(3)$ & $1(1)$ & GT28 & $1(0)$ & $5(0)$ \\
\hline GH89 & $3(3)$ & $1(0)$ & GT29 & 0 & $3(0)$ \\
\hline GH92 & $1(0)$ & 0 & GT30 & $1(0)$ & $1(0)$ \\
\hline GH95 & 0 & $1(0)$ & GT31 & $2(0)$ & $1(0)$ \\
\hline
\end{tabular}

Table 2 CAZy family enzymes in Goniomonas avonlea and Guillardia theta (those predicted to be secreted are in parentheses) (Continued) 
Table 2 CAZy family enzymes in Goniomonas avonlea and Guillardia theta (those predicted to be secreted are in parentheses) (Continued)

\begin{tabular}{|c|c|c|}
\hline CAZy families & Go. avonlea & Gu. theta \\
\hline GT32 & $5(0)$ & $9(0)$ \\
\hline GT33 & $1(0)$ & $1(0)$ \\
\hline GT34 & $2(0)$ & 0 \\
\hline GT35 & $2(0)$ & $2(0)$ \\
\hline GT37 & $3(0)$ & $1(0)$ \\
\hline GT39 & $1(0)$ & $1(0)$ \\
\hline GT41 & $88(0)$ & $60(8)$ \\
\hline GT47 & $2(0)$ & $1(0)$ \\
\hline GT48 & $1(0)$ & 0 \\
\hline GT49 & $7(0)$ & $19(0)$ \\
\hline GT50 & $1(0)$ & $1(0)$ \\
\hline GT54 & $1(0)$ & $1(0)$ \\
\hline GT57 & $3(0)$ & $2(0)$ \\
\hline GT58 & $5(0)$ & $1(0)$ \\
\hline GT59 & $1(0)$ & $1(0)$ \\
\hline GT60 & $1(0)$ & $1(0)$ \\
\hline GT61 & $5(0)$ & $4(0)$ \\
\hline GT64 & 0 & $2(1)$ \\
\hline GT66 & $7(0)$ & $3(0)$ \\
\hline GT68 & 0 & $1(0)$ \\
\hline GT69 & 0 & $1(0)$ \\
\hline GT71 & $1(0)$ & $1(0)$ \\
\hline GT74 & $2(0)$ & $2(0)$ \\
\hline GT75 & $1(0)$ & $1(0)$ \\
\hline GT76 & $1(0)$ & $1(0)$ \\
\hline GT77 & $1(0)$ & $3(0)$ \\
\hline GT90 & $5(0)$ & $2(0)$ \\
\hline GT96 & $8(0)$ & $2(0)$ \\
\hline CBM50 & $1(0)$ & $4(2)$ \\
\hline CBM73 & $2(0)$ & 0 \\
\hline
\end{tabular}

Abbreviations: $G H$ glycoside hydrolase, GT glycosyltransferase, CBM carbohydrate-binding module

analysis of the CAZomes of both organisms and compared them to those of other eukaryotes. On the basis of similarity clustering (Additional file 2: Figure S14), we observed a close relationship between the GT families of $G u$. theta and Go. avonlea. However, when the GH profiles are compared (Additional file 2: Figure S15), Go. avonlea is more similar to the rotifer Adineta vaga, an organism known to be able to degrade cellulose [89], and Gu. theta is closer to metazoans. When the CAZome as a whole is analyzed, i.e., all of the carbohydrate-active enzymes predicted for each organism, the Go. avonlea profile is most similar to that of Gu. theta (Fig. 7) and, together, these two cryptomonads are generally similar to other organisms with secondarily derived plastids. To determine whether the link between the CAZome profiles of Go. avonlea and plastid-bearing organisms is simply due to the presence of Gu. theta, we removed the latter organism and repeated the clustering analysis (Additional file 2: Figure S16). Even without Gu. theta, a specific relationship between the CAZomes of Go. avonlea and diverse algae (stramenopiles, Haptophyta, Rhizaria) is observed, suggesting that the carbohydrate-active enzyme profile of Go. avonlea is broadly similar to some algae (Additional file 2: Figure S16). In contrast, when Go. avonlea is removed from the analysis, Gu. theta was found to be closest to primary plastid-bearing algae, particularly Chlorophyta and Prasinophyta (Additional file 2: Figure S17). At the present time, the broader significance of these patterns is far from clear, but clearly the CAZomes-and carbohydrate metabolisms-of $\mathrm{Gu}$. theta and Go. avonlea are similar in some ways and different in others.

\section{From heterotroph to phototroph: the complexity of cryptomonads}

Subsequent to the evolution of primary plastids in Archaeplastida, a wide range of eukaryotes acquired photosynthesis secondarily via the engulfment of a red or green algal endosymbiont [2,4]. Despite more than a decade of cell biological, biochemical, phylogenetic, and phylogenomic investigation, it is still not clear how many times this occurred during the evolution of eukaryotes $[6,90,91]$. On the eukaryotic tree of life, secondary plastid-bearing organisms are scattered amongst plastid-lacking ones, and whether plastid gain or plastid loss has been the dominant mode of organelle evolution has proven difficult to discern.

In the case of red algal-derived plastids, recent evidence strongly suggests a single evolutionary origin of the plastid protein import machinery operating in cryptophytes, haptophytes, photosynthetic stramenopiles, and many alveolates: these organisms all use a host-derived multi-protein complex called SELMA (symbiont-specific endoplasmic reticulum-associated degradation-like machinery) for protein translocation across the second outermost plastid membrane of their four membrane-bound plastids [92-94] (the three membrane-bound plastids of dinoflagellates are an exception). This could mean that the plastids in each of these lineages stem from a single, ancient secondary endosymbiosis involving a red alga and a heterotrophic host (the so-called "chromalveolate" hypothesis [91]), followed by extensive plastid loss in their heterotrophic relatives. Alternatively, one or more cryptic tertiary endosymbioses could have spread the original secondary red plastid (and the genes for SELMA proteins) 


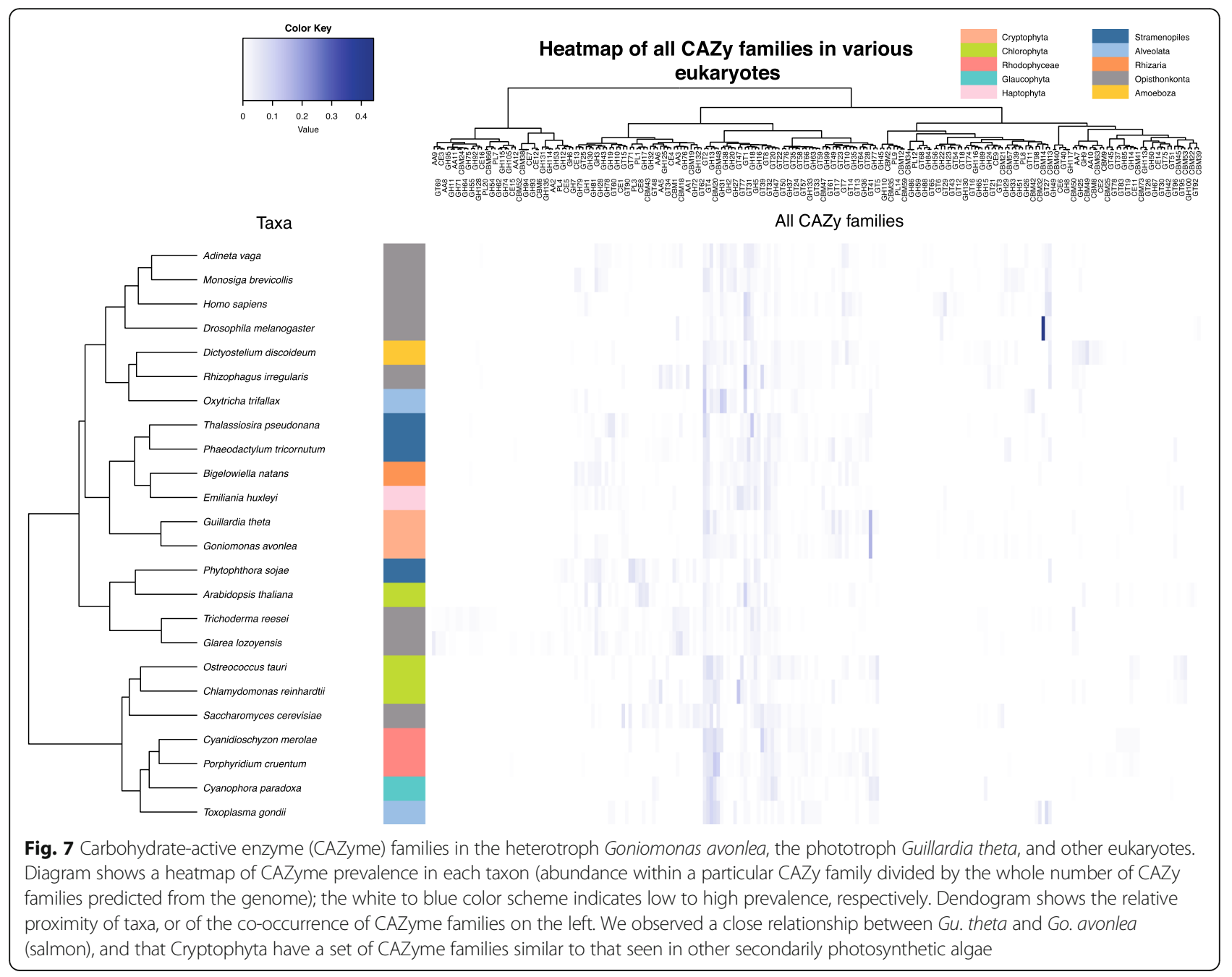

across the eukaryotic tree (see, e.g., [80, 95, 96]). Distinguishing between these two scenarios is fraught with challenges, not least of which is the fact that the deep structure of the eukaryotic tree of life continues to evolve (see above; Fig. 5) and the biology of many heterotrophic protist lineages remains poorly described.

Using genomic and transcriptomic data, we have explored the metabolic capacities of Go. avonlea-the first member of the heterotrophic Goniomonadea to have its genome sequenced-and compared them to those of the model plastid-bearing cryptophyte Gu. theta. We have shown that in Gu. theta, endosymbiosis led to the gain of metabolic pathways/processes presumably already present in the host, such as fatty acid biosynthesis, as well as de novo acquisition of photosynthesis and the MEP/DOXP pathway (Additional file 2: Figure S5 and S6). Despite its apparent redundancy with the cytosolic mevalonate (MVA) pathway, the plastid-localized MEP/ DOXP pathway in Gu. theta and other cryptophytes is presumably "useful" given that its end product feeds into other metabolic pathways. For example, the MEP/DOXP pathway generates phytyl-PP, which is used to produce several important components for photosynthesis such as phylloquinone, tocopherol, and chlorophyll [97-99]. Some organisms use the plastid MEP/DOXP pathway instead of the cytosolic MVA pathway, e.g., chlorophyte algae and certain dinoflagellates [100]. In Gu. theta, the benefits of photosynthesis presumably offset the costs associated with partial or complete metabolic redundancies. Nevertheless, Gu. theta does appear to have lost certain metabolic capacities that were present in its heterotrophic ancestors, such as primary bile biosynthesis (Additional file 2: Figure S4 and S11).

Glycosyl transferases (GTs) catalyze the transfer of sugars from donor to acceptor molecules, thereby creating glycosidic bonds. The evolution of photosynthesis is generally associated with the acquisition of new GT families and this is indeed what seems to have happened in the cryptophytes. Interestingly, however, we did not observe a significant increase in the total number of genes for GT enzymes in Gu. theta relative to Go. avonlea, and in fact, some GTs are present in Go. avonlea but 


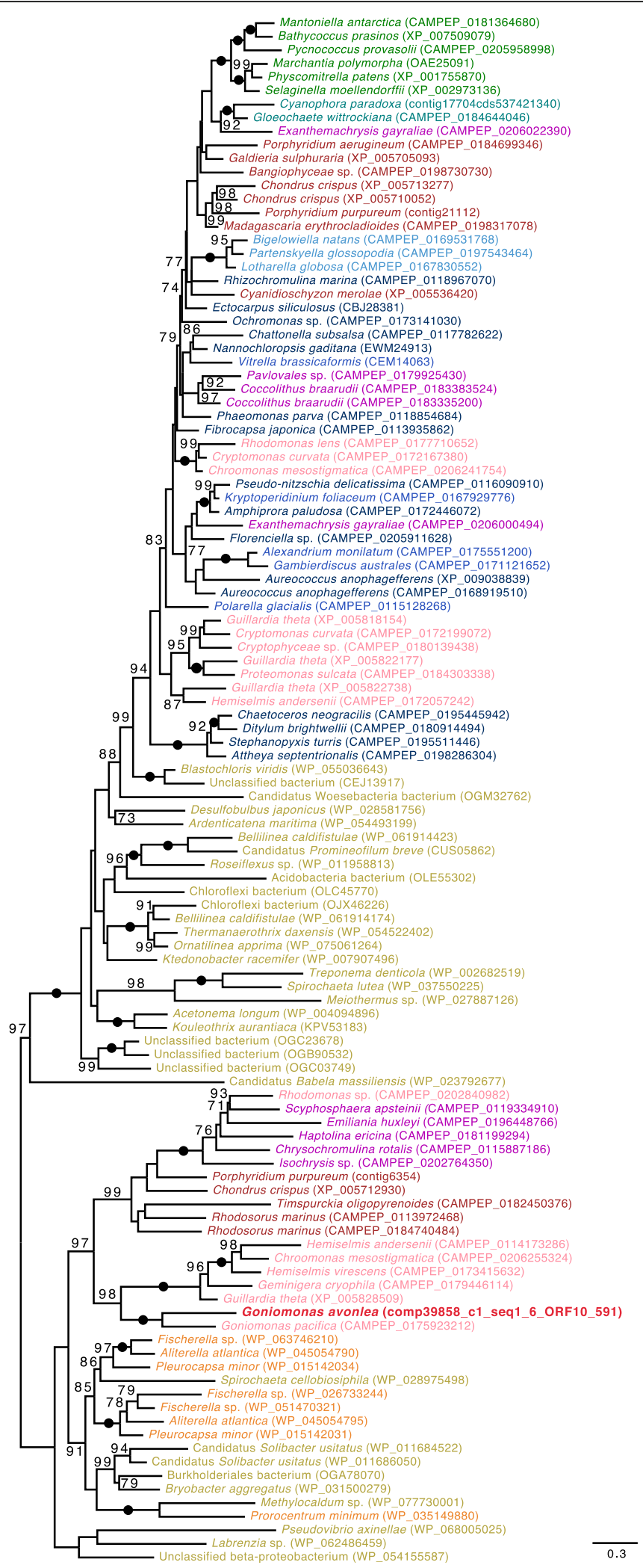

Fig. 8 (See legend on next page.) 
(See figure on previous page.)

Fig. 8 Phylogenetic analysis of glycosyltransferase (GT) 28. The tree shown is a maximum likelihood tree with ultrafast bootstrap values mapped onto the nodes. The tree shown is midpoint rooted. Sequences are colored according to their taxonomic affiliation: Viridiplantae are in green, Glaucophyta are in turquoise, Rhodophyta are in dark red, Cyanobacteria are orange and other Bacteria are in gold, Cryptophyta are in pink, Goniomonas avonlea is dark red and bolded, Haptophyta are in purple, Stramenopiles are in dark blue, Alveolata are in blue, Rhizaria are in light blue. The GT28 from Go. avonlea groups with other cryptomonads and with Rhodophyta. It is noteworthy that GT28 grouping with Go. avonlea seem to be mitochondrial based on signal targeting prediction while GT28 on the upper part could be targeted to the plastid, based on targeting prediction signal. The scale bar shows the inferred number of amino acid substitutions per site

not in Gu. theta; some GTs (GT3, GT48, GT34) appear to have been lost in Gu. theta and others replaced (e.g., GT3 has probably been replaced by a GT5 in Gu. theta) [82]. In addition, the loss of GT48 seems to correlate with the loss of beta glucan synthesis, as Gu. theta appears capable of generating only alpha-glucan polysaccharides, as in the red algal progenitor of its plastid.

Most unexpected was the discovery of a sequence of the GT28 family in Go. avonlea (Table 2), a category of enzymes known to be involved in the synthesis of cell wall components in bacteria (MurG is a GT28 enzyme acting as a UDP- $N$-acetylglucosamine:lipopolysaccharide $N$-acetylglucosamine transferase) and the synthesis of galactoglycerolipids in plastid-bearing organisms (MGD is a GT28 enzyme synthesizing 1,2-Diacyl-3-beta-Dgalactosyl-sn-glycerol). All GT28-containing eukaryotes in the public CAZy database have plastids (http:// www.cazy.org/GT28_eukaryota.html), and more recent investigation shows that of the more than 1000 eukaryotes that have had their CAZomes annotated, only eight have GT28 genes and lack plastids (most of these are fungi; data not shown). The GT28 gene in Go. avonlea resides on a contig with six other genes, five of which have a top blast hit to another eukaryote (2 being $G u$. theta), and the sixth is a bacterial-like gene that also has a close homolog in the Gu. theta nuclear genome (Additional file 2: Figure S18). In addition, the GT28 gene in Go. avonlea contains spliceosomal introns, confirming its provenance as a eukaryotic nuclear gene. The exact function of the Go. avonlea GT28 enzyme is difficult to predict with confidence. Based on sequence similarity, it could be a 1,2-diacylglycerol 3-beta-galactosy ltransferase [EC 2.4.1.46] and is predicted to be targeted to the mitochondrion by TargetP and PredSL; this is also thought to be the case in some non-photosynthetic algae [101]. We speculate that the Go. avonlea enzyme might be involved in the synthesis of phosphate-free mitochondrial lipids, which could serve in a phospholi pid-to-galactolipid exchange as observed in Arabidopsis thaliana mitochondria during phosphate starvation [102]. The origin and function of the GT28 gene in Go. avonlea thus appears distinct from the MGD genes of plastid-bearing eukaryotes. Biochemical, physiological, and ideally functional genomic studies need to be undergone to solve this question. Regardless of its precise function, it is noteworthy that in phylogenetic analyses, the GT28 homologs of Go. avonlea and Go. pacifica branch robustly with cryptophytes (including Gu. theta), red algae, and haptophytes (Fig. 8).

Another unexpected carbohydrate-active enzyme in Go. avonlea is glucan water dikinase (GWD). The discovery of GWD in a goniomonad is unexpected because it has long been assumed that Gu. theta and other cryptophytes acquired starch metabolism as a result of the acquisition of its secondary plastid. GWD has thus far only been found in organisms known to accumulate starch; the enzyme has been proposed to have evolved concomitantly with the primary plastid found in Viridiplantae, Rhodophyta, and Glaucophyta (i.e., Archaeplastida) [103]. Indeed, all eukaryotic starch accumulators either have a plastid or have been proposed to have once had a plastid — such as ciliates [104] —at some point during their evolution [82, 103] (Fig. 9). In Go. avonlea, the GWD gene contains introns and resides on a contig with seven other genes, three of which are clearly of eukaryotic provenance (Additional file 2: Figure S19). Given the absence of obvious starch granules in Go. avonlea, it is possible that the organism only synthesizes starch at certain stages of its life cycle, as is the case in the apicomplexan Toxoplasma gondii [105]. In phylogenetic analyses, the Go. avonlea GWD homolog does not branch with cryptophytes, but rather is weakly associated with a clade containing sequences from Rhodophyta, Glaucophyta, and various algae with red algal type plastids. How Go. avonlea came to possess and retain its GWD gene is an open question.

\section{Conclusions}

We have sequenced the nuclear genome of Goniomonas avonlea, the first of its kind for a plastid-lacking cryptomonad and only the second to be sequenced for all of Cryptista. The Go. avonlea genome provides a much-needed first glimpse into the biology of a heterotrophic protist residing on a large, poorly understood branch of the eukaryotic tree. Amongst the 33,470 predicted protein-coding genes in the Go. avonlea genome are hundreds of genes for carbohydrate-active enzymes that provide important clues as to what this phagotrophic protist eats in nature-the organism appears capable of digesting bacteria as well as eukaryotes, including algae. We found no convincing phylogenetic 


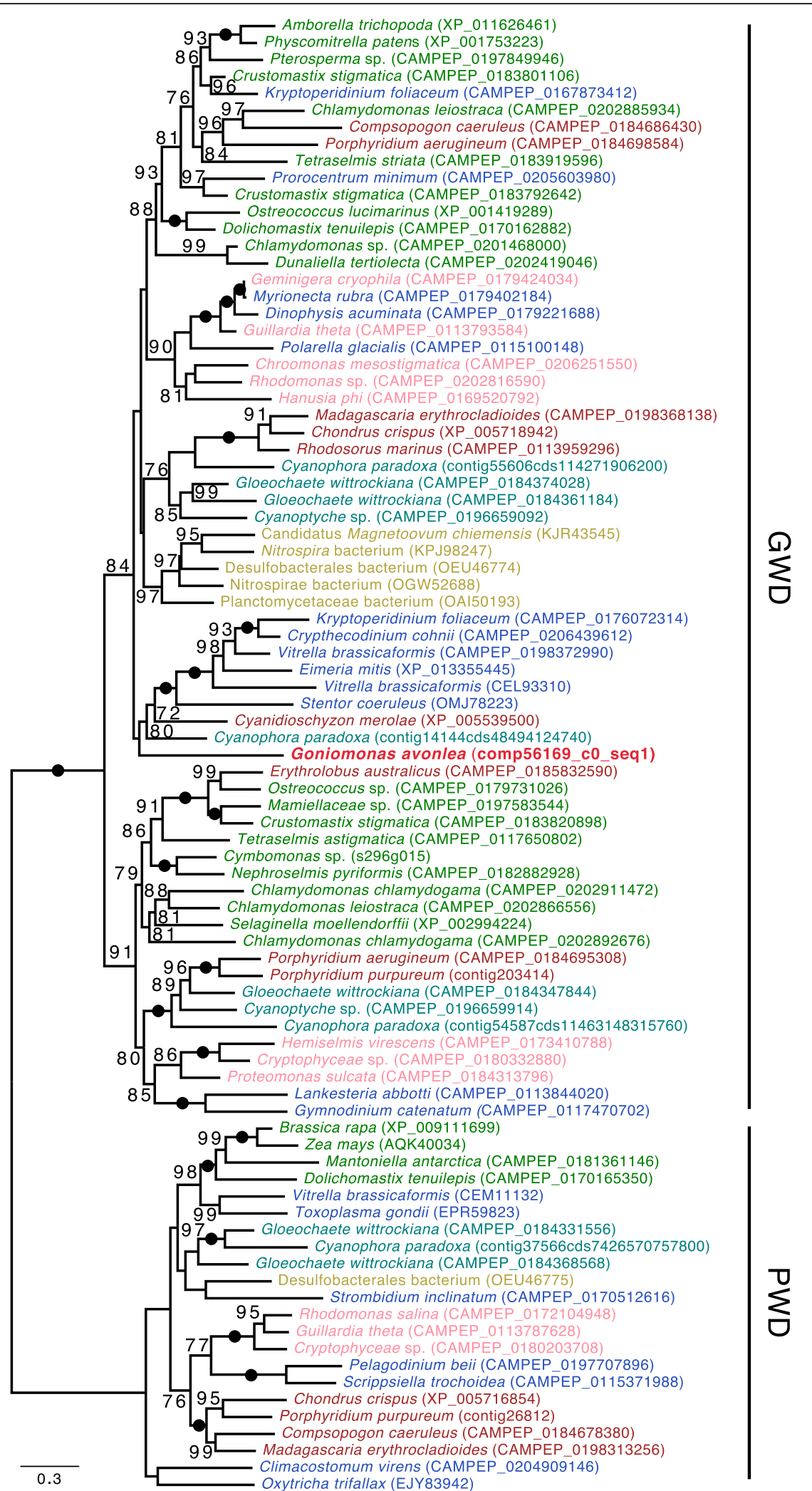

Fig. 9 (See legend on next page.) 
(See figure on previous page.)

Fig. 9 Glucan Water Dikinase (GWD) phylogenetic tree. The tree shown is a maximum likelihood tree with ultrafast bootstrap values mapped onto the nodes. The tree is rooted with the Phosphoglucan dikinase (PWD) sequences. Sequences are colored according to their taxonomic affiliation: Viridiplantae are in green, Glaucophyta are in turquoise, Rhodophyta are in dark red, Cyanobacteria are orange and other Bacteria are in gold, Cryptophyta are in pink, Goniomonas avonlea is dark red and bolded, Haptophyta are in purple, Stramenopiles are in dark blue, Alveolata are in blue, Rhizaria are in light blue. Some bacteria (in gold) could have obtained their GWD gene by LGT. The GWD homolog from Go. avonlea branches close to its counterpart in Rhodophyta and Glaucophyta; GWDs in Cryptophyta appear more distantly related. The scale bar shows the inferred number of amino acid substitutions per site

evidence to support the notion that Go. avonlea evolved from a secondary plastid-bearing ancestor; in terms of abundance, the handful of "algal" genes in the genome do not rise above background "noise." Nevertheless, this enigmatic protist possesses genes for enzymes such as GT28 and GWD, which are almost invariably found in plastid-bearing organisms. This is interesting for various reasons, not least of which is the fact that analysis of a 250-protein dataset placed cryptophytes, goniomonads (including Go. avonlea), and other heterotrophic Cryptista within the primary plastid-bearing Archaeplastida. While Go. avonlea serves as an important reference point for studying the metabolic transformation that took place during secondary endosymbiosis in the ancestor of modern-day Cryptophyceae, aspects of biochemistry and molecular biology may be linked to its deep ties with primary plastid-bearing organisms. More genomic data from diverse heterotrophic members of the Cryptista will hopefully allow us to test this hypothesis.

\section{Additional files}

Additional file 1: Flowchart summarizing sub-cellular localization predictions for Goniomonas avonlea proteins. (PDF $95 \mathrm{~kb}$ )

Additional file 2: Table S1. BUSCO analysis of Goniomonas avonlea, Guillardia theta, and Dictyostelium discoideum proteomes. Figure S1. Top: mitochondrial genome of Goniomonas avonlea. Bottom: Gene presence/ absence matrix for Cryptophyta and other eukaryotes. Figure S2. KEGG map of the metabolic pathways in Go. avonlea compared to plastid/ periplastidal pathways in Gu. theta. Figure S3. KEGG map of metabolic pathways in a putative secondary plastid in Go. avonlea compared to plastid/periplastidal pathways in Gu. theta and Arabidopsis. Figure S4. Metabolic maps for Go. avonlea and Gu. theta. Figure S5.-S13. KEGG representation of photosynthesis (Figure S5), terpenoid backbone biosynthesis (Figure S6.), carotenoid biosynthesis (Figure S7.), porphyrin and chlorophyll metabolism (Figure S8.), ubiquinone biosynthesis (Figure S9.), thiamine metabolism (Figure S10.), primary bile acid biosynthesis (Figure S11.), cellular metabolism (Figure S12.), and fatty acid biosynthesis (Figure S13.) in Go. avonlea. Figure S14. Comparison of GlycosylTransferase (GT) CAZy families in Go. avonlea and other eukaryotes. Figure S15. Comparison of Glycoside Hydrolase CAZy families in Go. avonlea and other eukaryotes. Figure S16. Comparison of all CAZy families in Go. avonlea and other eukaryotes. Figure S17. CAZy prevalence in various eukaryotes. Figure S18. Genomic context of GT28 in Go. avonlea. Figure S19. Genomic context of glucan water dikinase (GWD) in Go. avonlea. (PDF $11691 \mathrm{~kb}$ )

\section{Acknowledgements}

We thank two anonymous reviewers for helpful comments on an earlier version of this manuscript.

\section{Funding}

The Archibald Lab acknowledges support in the form of a Discovery grant (RGPIN-2014-05871) from the Natural Sciences and Engineering Research Council of Canada. EK is the recipient of a Simons Foundation award (SF382790). EM is supported by the French National Research Agency (ANR-10LABEX-04 GRAL Labex, Grenoble Alliance for Integrated Structural Cell Biology; ANR-11-BTBR-0008 Océanomics). SJS was supported by a NSERC CGS-M graduate scholarship and a Nova Scotia Graduate Scholarship.

\section{Availability of data and materials}

The genomic and transcriptomic datasets supporting the conclusions of this article are available in GenBank under accession numbers QUTJ00000000 and GGUN00000000, respectively, while the Go. avonlea mitochondrial genome is available under the following accession number: AP018919.

\section{Authors' contributions}

EK and JMA designed the study. UC, SJS, BAC, RK, LE, DM, BH, EM, MC, and CD collected data and carried out analyses; all authors contributed to data interpretation; and UC, SJS, BAC, EK, and JMA wrote and revised the manuscript. All authors read and approved the final manuscript.

Ethics approval and consent to participate

Not applicable.

Competing interests

The authors declare that they have no competing interests.

\section{Publisher's Note}

Springer Nature remains neutral with regard to jurisdictional claims in published maps and institutional affiliations.

\footnotetext{
Author details

${ }^{1}$ Department of Biochemistry and Molecular Biology, Dalhousie University, Halifax, Nova Scotia B3H 4R2, Canada. ${ }^{2}$ Centre for Comparative Genomics and Evolutionary Bioinformatics, Dalhousie University, Halifax, Nova Scotia, Canada. ${ }^{3}$ Graduate School of Human and Environmental Studies, Kyoto University, Kyoto, Kyoto 606-8501, Japan. ${ }^{4}$ Architecture et Fonction des Macromolécules Biologiques (AFMB), CNRS, Université Aix-Marseille, 163 Avenue de Luminy, 13288 Marseille, France. ${ }^{5}$ INRA, USC 1408 AFMB, 13288 Marseille, France. ${ }^{6}$ Department of Biological Sciences, King Abdulaziz

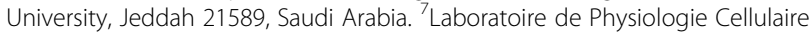
et Végétale, CNRS, CEA, INRA, Université Grenoble Alpes, Institut de Biosciences et Biotechnologies de Grenoble, CEA-Grenoble, 17 rue des Martyrs, 38000 Grenoble, France. ${ }^{8}$ Present address: UMR 8576 - Unité de glycobiologie structurale et fonctionnelle, Université Lille 1, 59650 Villeneuve d'Ascq, France. ${ }^{9}$ Canadian Institute for Advanced Research, Program in Integrated Microbial Biodiversity, Toronto, Ontario, Canada. ${ }^{10}$ Division of Invertebrate Zoology \& Sackler Institute for Comparative Genomics, American Museum of Natural History, Central Park West at 79 Street, New York, NY 10024, USA. ${ }^{11}$ Present address: Department of Cell and Molecular Biology, Science for Life Laboratory, Uppsala University, SE-75123 Uppsala, Sweden. ${ }^{12}$ Present address: Laboratory for Cell Biology, Philipps University Marburg, Karl-von-Frisch-Str. 8, 35043 Marburg, Germany.
} 


\section{Received: 4 August 2018 Accepted: 12 October 2018} Published online: 28 November 2018

\section{References}

1. Howe C, Barbrook A, Nisbet RE, Lockhart P, Larkum AW. The origin of plastids. Philos Trans R Soc B Biol Sci. 2008;363:2675-85.

2. Gould SB, Waller RF, McFadden GI. Plastid evolution. Annu Rev Plant Biol. 2008:59:491-517

3. Moreira D, Le Guyader H, Philippe $H$. The origin of red algae and the evolution of chloroplasts. Nature. 2000;405(6782):69-72.

4. Keeling PJ. The endosymbiotic origin, diversification and fate of plastids. Philos Trans R Soc B Biol Sci. 2010;365:729-48.

5. Adl SM, Simpson AGB, Lane CE, Lukeš J, Bass D, Bowser SS, et al. The revised classification of eukaryotes. J Eukaryot Microbiol. 2012;59:429-514.

6. Archibald JM. The puzzle of plastid evolution. Curr Biol. 2009;19:R81-8.

7. Kim E, Archibald JM. Ultrastructure and molecular phylogeny of the cryptomonad Goniomonas avonlea sp. nov. Protist. 2013;164:160-82.

8. Okamoto N, Chantangsi C, Horák A, Leander BS, Keeling PJ. Molecular phylogeny and description of the novel katablepharid Roombia truncata gen. Et sp. nov., and establishment of the Hacrobia taxon nov. PLoS One. 2009;4:e7080.

9. Cavalier-Smith T. Kingdom protozoa and its 18 phyla. Microbiol Rev. 1993; 57:953-94

10. Yabuki A, Kamikawa R, Ishikawa SA, Kolisko M, Kim E, Tanabe AS, et al. Palpitomonas bilix represents a basal cryptist lineage: insight into the character evolution in Cryptista. Sci Rep. 2014;4. https://doi.org/10.1038/ srep04641.

11. Patron NJ, Inagaki Y, Keeling PJ. Multiple gene phylogenies support the monophyly of cryptomonad and haptophyte host lineages. Curr Biol. 2007; 17:887-91.

12. Burki F, Inagaki Y, Bråte J, Archibald JM, Keeling PJ, Cavalier-Smith T, et al. Large-scale phylogenomic analyses reveal that two enigmatic protist lineages, telonemia and centroheliozoa, are related to photosynthetic chromalveolates. Genome Biol Evol. 2009;1:231-8.

13. Katz LA, Grant JR. Taxon-rich phylogenomic analyses resolve the eukaryotic tree of life and reveal the power of subsampling by sites. Syst Biol. 2015;64: 406-15

14. Burki F, Kaplan M, Tikhonenkov DV, Zlatogursky V, Minh BQ, Radaykina LV, et al. Untangling the early diversification of eukaryotes: a phylogenomic study of the evolutionary origins of Centrohelida, Haptophyta and Cryptista. Proc R Soc B Biol Sci. 2016:283:20152802.

15. Sibbald SJ, Archibald JM. More protist genomes needed. Nat Ecol Evol. 2017:1:0145.

16. Archibald JM. Nucleomorph genomes: structure, function, origin and evolution. BioEssays. 2007:29:392-402.

17. Moore CE, Curtis B, Mills T, Tanifuji G, Archibald JM. Nucleomorph genome sequence of the cryptophyte alga Chroomonas mesostigmatica CCMP1168 reveals lineage-specific gene loss and genome complexity. Genome Biol Evol. 2012;4:1162-75

18. Curtis BA, Tanifuji G, Burki F, Gruber A, Irimia M, Maruyama S, et al. Algal genomes reveal evolutionary mosaicism and the fate of nucleomorphs. Nature. 2012:492:59-65.

19. Rolland N, Curien G, Finazzi G, Kuntz M, Maréchal E, Matringe M, et al. The biosynthetic capacities of the plastids and integration between cytoplasmic and chloroplast processes. Annu Rev Genet. 2012;46:233-64.

20. Pfannschmidt T, Schutze K, Brost M, Oelmuller R. A novel mechanism of nuclear photosynthesis gene regulation by redox signals from the chloroplast during photosystem stoichiometry adjustment. J Biol Chem. 2001;276:36125-30.

21. Maruyama S, Suzaki T, Weber AP, Archibald JM, Nozaki H. Eukaryote-toeukaryote gene transfer gives rise to genome mosaicism in euglenids. BMC Evol Biol. 2011;11:105.

22. Lombard V, Golaconda Ramulu H, Drula E, Coutinho PM, Henrissat B. The carbohydrate-active enzymes database (CAZy) in 2013. Nucleic Acids Res. 2014;42:D490-5.

23. Okaichi T, Nishio S, Imatomi Y. Collection and mass culture. In: Yûdoku Purankuton-Hassei, Sayôkikô, Dokuseibun, editors. Toxic phytoplankton Occurence, mode of action and toxins. Tokyo:Jpn Fish Soc. 1982;22-34.

24. Bolger AM, Lohse M, Usadel B. Trimmomatic: a flexible trimmer for Illumina sequence data. Bioinformatics. 2014;30:2114-20.
25. Grabherr MG, Haas BJ, Yassour M, Levin JZ, Thompson DA, Amit I, et al. Fulllength transcriptome assembly from RNA-Seq data without a reference genome. Nat Biotechnol. 2011;29:644-52.

26. Butler J, MacCallum I, Kleber M, Shlyakhter IA, Belmonte MK, Lander ES, et al. ALLPATHS: De novo assembly of whole-genome shotgun microreads. Genome Res. 2008;18:810-20.

27. Simpson JT, Wong K, Jackman SD, Schein JE, Jones SJM, Birol I. ABySS: a parallel assembler for short read sequence data. Genome Res. 2009:19:1117-23.

28. Sommer DD, Delcher AL, Salzberg SL, Pop M. Minimus: a fast, lightweight genome assembler. BMC Bioinformatics. 2007;8:64

29. Boisvert S, Laviolette F, Corbeil J. Ray: simultaneous assembly of reads from a mix of high-throughput sequencing technologies. J Comput Biol. 2010;17: 1519-33.

30. Langmead B, Salzberg SL. Fast gapped-read alignment with bowtie 2. Nat Meth. 2012:9:357-9.

31. Clark SC, Egan R, Frazier PI, Wang Z. ALE: a generic assembly likelihood evaluation framework for assessing the accuracy of genome and metagenome assemblies. Bioinformatics. 2013;29:435-43.

32. Rahman A, Pachter L. CGAL: computing genome assembly likelihoods. Genome Biol. 2013;14:R8

33. Altschul SF, Madden TL, Schäffer AA, Zhang J, Zhang Z, Miller W, et al. Gapped BLAST and PSI-BLAST: a new generation of protein database search programs. Nucleic Acids Res. 1997;25:3389-402.

34. Stanke M, Diekhans M, Baertsch R, Haussler D. Using native and syntenically mapped cDNA alignments to improve de novo gene finding. Bioinformatics. 2008:24:637-44.

35. Stanke M, Schöffmann O, Morgenstern B, Waack S. Gene prediction in eukaryotes with a generalized hidden Markov model that uses hints from external sources. BMC Bioinformatics. 2006;7:62.

36. Haas BJ. Improving the Arabidopsis genome annotation using maximal transcript alignment assemblies. Nucleic Acids Res. 2003;31:5654-66.

37. Keeling P, Burki F, Wilcox J, Allam B, et al. The marine microbial eukaryote transcriptome sequencing project (MMETSP): illuminating the functional diversity of eukaryotic life in the oceans through transcriptome sequencing. PLoS Biol. 2014;12:e1001889.

38. Simão FA, Waterhouse RM, loannidis P, Kriventseva EV, Zdobnov EMBUSCO. Assessing genome assembly and annotation completeness with single-copy orthologs. Bioinformatics. 2015:31:3210-2.

39. Wang Y, Coleman-Derr D, Chen G, Gu YQ. OrthoVenn: a web server for genome wide comparison and annotation of orthologous clusters across multiple species. Nucleic Acids Res. 2015;43:W78-84

40. Wu S, Zhu Z, Fu L, Niu B, Li W. WebMGA: a customizable web server for fast metagenomic sequence analysis. BMC Genomics. 2011;12:444.

41. Mao X, Cai T, Olyarchuk JG, Wei L. Automated genome annotation and pathway identification using the KEGG Orthology (KO) as a controlled vocabulary. Bioinformatics. 2005;21:3787-93.

42. Kanehisa M, Sato Y, Morishima K. BlastKOALA and GhostKOALA: KEGG tools for functional characterization of genome and metagenome sequences. J Mol Biol. 2016:428:726-31.

43. Kanehisa M, Goto S, Sato Y, Furumichi M, Tanabe M. KEGG for integration and interpretation of large-scale molecular data sets. Nucleic Acids Res. 2012;40:D109-14.

44. Petsalaki El, Bagos PG, Litou ZL, Hamodrakas SJ. PredSL: a tool for the Nterminal sequence-based prediction protein subcellular localization. Genomics Proteomics Bioinformatics. 2006:4:48-55.

45. Emanuelsson O, Brunak S, von Heijne G, Nielsen H. Locating proteins in the cell using TargetP, SignalP and related tools. Nat Protoc. 2007;2:953-71.

46. Small I, Peeters N, Legeai F, Lurin C. Predotar: a tool for rapidly screening proteomes forN-terminal targeting sequences. Proteomics. 2004;4:1581-90.

47. Petersen TN, Brunak S, von Heijne G, Nielsen H. SignalP 4.0: discriminating signal peptides from transmembrane regions. Nat Methods. 2011:8:785-6.

48. Nielsen $\mathrm{H}$. Predicting secretory proteins with SignalP. Methods Mol Biol. 2017;1611:59-73.

49. Gruber A, Rocap G, Kroth PG, Armbrust EV, Mock T. Plastid proteome prediction for diatoms and other algae with secondary plastids of the red lineage. Plant J. 2015:81:519-28.

50. Mistry J, Finn RD, Eddy SR, Bateman A, Punta M. Challenges in homology search: HMMER3 and convergent evolution of coiled-coil regions. Nucleic Acids Res. 2013;41:e121.

51. Bray JR, Curtis JT. An ordination of the upland forest communities of southern Wisconsin. Ecol Monogr. 1957;27:325-49. 
52. Ward JH. Hierarchical grouping to optimize an objective function. J Am Stat Assoc. 1963;58:236.

53. Quinn GP, Keough MJ. Experimental design and data analysis for biologists. Cambridge: Cambridge University Press; 2002. https://doi.org/10.1017/ CBO9780511806384.

54. Soverini M, Turroni S, Biagi E, Quercia S, Brigidi P, Candela M, et al. Variation of carbohydrate-active enzyme patterns in the gut microbiota of Italian healthy subjects and type 2 diabetes patients. Front Microbiol. 2017;8. https://doi.org/10.3389/fmicb.2017.02079.

55. Oksanen J. Multivariate analysis of ecological communities in $\mathrm{R}$ : vegan tutorial. 2015. http://cc.oulu.fi/ jarioksa/opetus/metodi/vegantutor.pdf. Accessed 15 Dec 2015.

56. Buchfink B, Xie C, Huson DH. Fast and sensitive protein alignment using DIAMOND. Nat Methods. 2015;12:59-60.

57. Finn RD, Attwood TK, Babbitt PC, Bateman A, Bork P, Bridge AJ, et al. InterPro in 2017-beyond protein family and domain annotations. Nucleic Acids Res. 2017:45:D190-9.

58. Katoh K, Standley DM. MAFFT multiple sequence alignment software version 7: improvements in performance and usability. Mol Biol Evol. 2013;30:772-80.

59. Criscuolo A, Gribaldo S. BMGE (block mapping and gathering with entropy): a new software for selection of phylogenetic informative regions from multiple sequence alignments. BMC Evol Biol. 2010;10:210.

60. Price MN, Dehal PS, Arkin AP. Others. FastTree 2-approximately maximumlikelihood trees for large alignments. PLoS One. 2010;5:e9490.

61. Nguyen L-T, Schmidt HA, von Haeseler A, Minh BQ. IQ-TREE: a fast and effective stochastic algorithm for estimating maximum-likelihood phylogenies. Mol Biol Evol. 2015;32:268-74.

62. Le SQ, Dang CC, Gascuel O. Modeling protein evolution with several amino acid replacement matrices depending on site rates. Mol Biol Evol. 2012;29: 2921-36.

63. Minh BQ, Nguyen MAT, von Haeseler A. Ultrafast approximation for phylogenetic bootstrap. Mol Biol Evol. 2013;30:1188-95.

64. Cenci U, Moog D, Curtis BA, Tanifuji G, Eme L, Lukeš J, et al. Heme pathway evolution in kinetoplastid protists. BMC Evol Biol. 2016;16. https://doi.org/10. 1186/s12862-016-0664-6.

65. Cenci U, Ducatez M, Kadouche D, Colleoni C, Ball SG. Was the chlamydial adaptative strategy to tryptophan starvation an early determinant of plastid endosymbiosis? Front Cell Infect Microbiol. 2016;6. https://doi.org/10.3389/ fcimb.2016.00067.

66. Maruyama S, Eveleigh RJ, Archibald JM. Treetrimmer: a method for phylogenetic dataset size reduction. BMC Res Notes. 2013;6:145.

67. Edgar RC. MUSCLE: multiple sequence alignment with high accuracy and high throughput. Nucleic Acids Res. 2004;32:1792-7.

68. Burki F, Kaplan M, Tikhonekov DV, Zlatogursky V, Minh BQ, Radaykina LV, et al. Data from: Untangling the early diversification of eukaryotes: a phylogenomic study of the evolutionary origins of Centrohelida, Haptophyta, and Cryptista. 2016. doi:https://doi.org/10.5061/dryad.rj87v.

69. Wang H-C, Minh BQ, Susko E, Roger AJ. Modeling site heterogeneity with posterior mean site frequency profiles accelerates accurate phylogenomic estimation. Syst Biol. 2018;67:216-35.

70. Kalyaanamoorthy S, Minh B, Wong T, von Haeseler A, Jermiin L. ModelFinder: fast model selection for accurate phylogenetic estimates. Nat Methods. 2017;14:587-9.

71. de Vienne DM, Ollier S, Aguileta G. Phylo-MCOA: a fast and efficient method to detect outlier genes and species in phylogenomics using multiple coinertia analysis. Mol Biol Evol. 2012;29:1587-98.

72. Kim E, Lane CE, Curtis BA, Kozera C, Bowman S, Archibald JM. Complete sequence and analysis of the mitochondrial genome of Hemiselmis andersenii CCMP644 (Cryptophyceae). BMC Genomics. 2008;9:215.

73. Kim J, Yoon HS, Yi G, Shin W, Archibald JM. Comparative mitochondrial genomics of cryptophyte algae: gene shuffling and dynamic mobile genetic elements. BMC Genomics. 2018;19. https://doi.org/10.1186/s12864-018-4626-9.

74. Nishimura Y, Tanifuji G, Kamikawa R, Yabuki A, Hashimoto T, Inagaki Y. Mitochondrial genome of Palpitomonas bilix : derived genome structure and ancestral system for cytochrome c maturation. Genome Biol Evol. 2016; 8:3090-8.

75. McFadden Gl, Gilson PR, Hill DRA. Goniomonas: rRNA sequences indicate that this phagotrophic flagellate is a close relative of the host component of cryptomonads. Eur J Phycol. 1994;29:29-32.

76. Martin-Cereceda M, Roberts EC, Wootton EC, Bonaccorso E, Dyal P, Guinea A, et al. Morphology, ultrastructure, and small subunit rDNA phylogeny of the marine heterotrophic flagellate Goniomonas aff. amphinema. J Eukaryot Microbiol. 2010;57:159-70.

77. Stiller JW, Huang J, Ding Q, Tian J, Goodwillie C. Are algal genes in nonphotosynthetic protists evidence of historical plastid endosymbioses? BMC Genomics. 2009;10:484.

78. Cavalier-Smith T. Symbiogenesis: mechanisms, evolutionary consequences, and systematic implications. Annu Rev Ecol Evol Syst. 2013;44:145-72.

79. Heiss AA, Kolisko M, Ekelund F, Brown MW, Roger AJ, Simpson AGB. Combined morphological and phylogenomic re-examination of malawimonads, a critical taxon for inferring the evolutionary history of eukaryotes. R Soc Open Sci. 2018;5:171707.

80. Archibald JM. Endosymbiosis and eukaryotic cell evolution. Curr Biol. 2015; 25:R911-21.

81. Burki F, Okamoto N, Pombert J-F, Keeling PJ. The evolutionary history of haptophytes and cryptophytes: phylogenomic evidence for separate origins. Proc R Soc B Biol Sci. 2012;279:2246-54.

82. Ball S, Colleoni C, Cenci U, Raj JN, Tirtiaux C. The evolution of glycogen and starch metabolism in eukaryotes gives molecular clues to understand the establishment of plastid endosymbiosis. J Exp Bot. 2011;62:1775-801.

83. O'Neill EC, Trick M, Hill L, Rejzek M, Dusi RG, Hamilton CJ, et al. The transcriptome of Euglena gracilis reveals unexpected metabolic capabilities for carbohydrate and natural product biochemistry. Mol BioSyst. 2015;11:2808-20.

84. Popper ZA, Michel G, Hervé C, Domozych DS, Willats WGT, Tuohy MG, et al. Evolution and diversity of plant cell walls: from algae to flowering plants. Annu Rev Plant Biol. 2011;62:567-90.

85. Burns JA, Pittis AA, Kim E. Gene-based predictive models of trophic modes suggest Asgard archaea are not phagocytotic. Nat Ecol Evol. 2018;2:697-704.

86. McKie-Krisberg ZM, Gast RJ, Sanders RW. Physiological responses of three species of antarctic mixotrophic phytoflagellates to changes in light and dissolved nutrients. Microb Ecol. 2015;70:21-9.

87. Selosse M-A, Charpin M, Not F. Mixotrophy everywhere on land and in water: the grand écart hypothesis. Ecol Lett. 2017;20:246-63.

88. Tranvik LJ, Porter KG, Sieburth JM. Occurrence of bacterivory in Cryptomonas, a common freshwater phytoplankter. Oecologia. 1989;78:473-6.

89. Flot J-F, Hespeels B, Li X, Noel B, Arkhipova I, Danchin EGJ, et al. Genomic evidence for ameiotic evolution in the bdelloid rotifer Adineta vaga. Nature. 2013;500:453-7.

90. Zimorski V, Ku C, Martin WF, Gould SB. Endosymbiotic theory for organelle origins. Curr Opin Microbiol. 2014;22:38-48.

91. Cavalier-Smith T. Principles of protein and lipid targeting in secondary symbiogenesis: euglenoid, dinoflagellate, and sporozoan plastid origins and the eukaryote family tree. J Eukaryot Microbiol. 1999;46:347-66.

92. Hempel F, Bullmann L, Lau J, Zauner S, Maier UG. ERAD-derived preprotein transport across the second outermost plastid membrane of diatoms. Mol Biol Evol. 2009;26:1781-90.

93. Sommer MS, Gould SB, Lehmann P, Gruber A, Przyborski JM, Maier U-G. Der1-mediated preprotein import into the periplastid compartment of chromalveolates? Mol Biol Evol. 2007;24:918-28.

94. Stork S, Moog D, Przyborski JM, Wilhelmi I, Zauner S, Maier UG. Distribution of the SELMA translocon in secondary plastids of red algal origin and predicted uncoupling of ubiquitin-dependent translocation from degradation. Eukaryot Cell. 2012;11:1472-81.

95. Stiller JW, Schreiber J, Yue J, Guo H, Ding Q, Huang J. The evolution of photosynthesis in chromist algae through serial endosymbioses. Nat Commun. 2014;5:5764.

96. Baurain D, Brinkmann H, Petersen J, Rodriguez-Ezpeleta N, Stechmann A, Demoulin $V$, et al. Phylogenomic evidence for separate acquisition of plastids in cryptophytes, haptophytes, and stramenopiles. Mol Biol Evol. 2010;27:1698-709.

97. Gross J, Cho WK, Lezhneva L, Falk J, Krupinska K, Shinozaki K, et al. A plant locus essential for phylloquinone (vitamin K1) biosynthesis originated from a fusion of four eubacterial genes. J Biol Chem. 2006;281:17189-96.

98. Emonds-Alt B, Coosemans N, Gerards T, Remacle C, Cardol P. Isolation and characterization of mutants corresponding to the MENA, MENB, MENC and MENE enzymatic steps of 5'-monohydroxyphylloquinone biosynthesis in Chlamydomonas reinhardtii. Plant J. 2016. https://doi.org/10.1111/tpj.13352.

99. Chatterjee A, Kundu S. Revisiting the chlorophyll biosynthesis pathway using genome scale metabolic model of Oryza sativa japonica. Sci Rep. 2015;5:14975.

100. Bentlage B, Rogers TS, Bachvaroff TR, Delwiche CF. Complex ancestries of isoprenoid synthesis in dinoflagellates. J Eukaryot Microbiol. 2016;63:123-37. 
101. Borza T, Popescu CE, Lee RW. Multiple metabolic roles for the nonphotosynthetic plastid of the green alga Prototheca wickerhamii. Eukaryot Cell. 2005;4:253-61.

102. Michaud M, Gros V, Tardif M, Brugière $S$, Ferro M, Prinz WA, et al. AtMic60 is involved in plant mitochondria lipid trafficking and is part of a large complex. Curr Biol. 2016;26:627-39.

103. Cenci U, Nitschke F, Steup M, Minassian BA, Colleoni C, Ball SG. Transition from glycogen to starch metabolism in Archaeplastida. Trends Plant Sci. 2014;19:18-28

104. Reyes-Prieto A, Moustafa A, Bhattacharya D. Multiple genes of apparent algal origin suggest ciliates may once have been photosynthetic. Curr Biol. 2008;18:956-62.

105. Coppin A, Varré J-S, Lienard L, Dauvillée D, Guérardel Y, Soyer-Gobillard M$\mathrm{O}$, et al. Evolution of plant-like crystalline storage polysaccharide in the protozoan parasite Toxoplasma gondii argues for a red alga ancestry. J Mol Evol. 2005;60:257-67.

Ready to submit your research? Choose BMC and benefit from:

- fast, convenient online submission

- thorough peer review by experienced researchers in your field

- rapid publication on acceptance

- support for research data, including large and complex data types

- gold Open Access which fosters wider collaboration and increased citations

- maximum visibility for your research: over $100 \mathrm{M}$ website views per year

At BMC, research is always in progress.

Learn more biomedcentral.com/submissions 